\title{
Developing horizontal convection against stable temperature stratification in a rectangular container
}

\author{
Daisuke Noto $\odot,{ }^{1, *}$ Tomomi Terada $\odot,{ }^{1}$ Takatoshi Yanagisawa $\odot,{ }^{2,1}$ \\ Takehiro Miyagoshi $\odot,^{2}$ and Yuji Tasaka $\odot^{1}$ \\ ${ }^{1}$ Laboratory for Flow Control, Faculty of Engineering, Hokkaido University, Sapporo, Japan \\ ${ }^{2}$ Japan Agency for Marine-Earth Science and Technology (JAMSTEC), Yokosuka, Japan
}

(Received 29 January 2021; accepted 29 July 2021; published 25 August 2021)

\begin{abstract}
The effect of background stable temperature stratification in the developing stage of horizontal convection is studied by conducting laboratory experiments. By imposing horizontally differential heating at the top of a layer of low-temperature water, both vertical and horizontal temperature differences are explicitly defined. In developing horizontal convection of the present study, the flow structures are driven only by the baroclinic torque produced by the horizontal temperature difference, and braked by the restoring force of stable temperature stratification. We thus defined a nondimensional stratification parameter, which represents the balance of the braking force and the baroclinic torque, in addition to the Rayleigh number. Various features of the flow structures, maximum velocity, stream function, roll thickness, circulation of the roll, total kinetic energy, and Reynolds number, which are quantified via particle-tracking velocimetry, are summarized in spaces of the two nondimensional parameters. In the developing horizontal convection, the quantified flow features are well organized by power laws of the nondimensional parameters. The finite domain of the fluid container augments the effect of the apparent braking force, and the bulk quantities of the roll structures are suppressed by the stable temperature stratification. These results are the evidences for the significance of the nondimensional stratification parameter in the developing horizontal convection, unlike thermally equilibrated horizontal convection conventionally considering destabilizing thermal buoyancy as the primary driving force.
\end{abstract}

DOI: 10.1103/PhysRevFluids.6.083501

\section{INTRODUCTION}

Horizontal convection (HC), driven by a temperature gradient along a horizontal surface at the same level, is a fundamental topic describing fluid motions with various length scales in geophysical flows and engineering processes. Flows originating from $\mathrm{HC}$ are universal and have thus interest in research on meridional overturning circulation in ocean [1-7], land- and sea-breeze circulation, heat- and cool-island circulation [8-11], and glass-melting furnaces [12,13]. Extensive reviews and detailed discussions of HC can be found in Hughes and Griffiths [14] and references therein.

Conventionally, $\mathrm{HC}$ has been studied in its thermally equilibrated state as illustrated in Fig. 1(a). Here we consider a situation that different temperatures, a high-temperature $T_{\mathrm{h}}$ and a lowtemperature $T_{1}$, are imposed on the top surface of the fluid layer with an internal temperature $T_{\mathrm{i}}$ confined by adiabatic walls, resulting in $T_{1}<T_{\mathrm{i}}<T_{\mathrm{h}}$. One-half of the top surfaces act as heating and the other as cooling boundaries to the fluid layer; the heat flux to the fluid is balanced in the thermally equilibrated state [15-19]. The two horizontal boundaries with different temperatures are

*noto@ring-me.eng.hokudai.ac.jp 

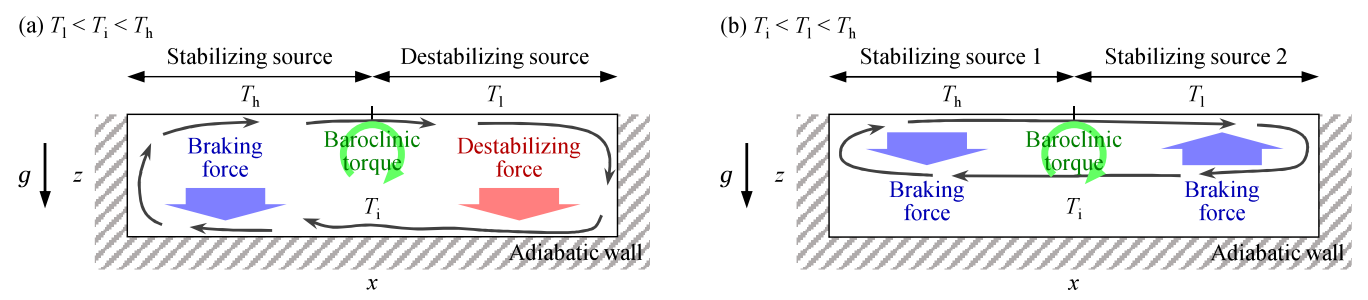

FIG. 1. Conceptual diagrams of two different problem settings for HC: (a) thermally equilibrated HC and (b) developing HC. $T_{\mathrm{i}}$ denotes the interior temperature, and $T_{\mathrm{h}}$ and $T_{1}$ are the temperatures set at the same horizontal plane, $T_{\mathrm{h}}>T_{1}$.

thus respectively worked as a "stabilizing source" and "destabilizing source" [14] to the internal fluid of $T_{\mathrm{i}}$. Convective motion is then driven by the baroclinic torque produced by the horizontal temperature difference, and a clockwise circulation is formed as illustrated in Fig. 1(a). The thermal buoyancy at the destabilizing source also drives the circulation, as the destabilizing source is always thermally unstable as long as $T_{1}<T_{\mathrm{i}}$, irrespective of the presence of the horizontal temperature difference. In addition to these two effects facilitating the convective motion, the braking force originated from the restoring force of the stable temperature stratification at the stabilizing source decelerates the clockwise circulation. As a result, an asymmetric convection structure is generated. For most cases, a single overturning convection cell penetrating full height of the fluid layer is formed as illustrated by black arrows in Fig. 1(a). Since thermal buoyancy explicitly facilitates this thermally equilibrated HC, various characteristic quantities, such as features of convection cell and heat transport in terms of the Nusselt number, have been described using the Rayleigh number, like those in Rayleigh-Bénard convection (see, e.g., [1,5,13,17,20,21]). Here the Rayleigh number meaning a ratio of thermal buoyancy potentially existing in the bulk to the viscous and thermal dissipation is represented as

$$
\mathrm{Ra}=\frac{g \beta \Delta \theta W^{3}}{\kappa \nu},
$$

where $g, \beta, v, \kappa, \Delta \theta$, and $W$ are, respectively, the gravity acceleration, thermal expansion coefficient, kinematic viscosity, thermal diffusivity, horizontal temperature difference, and horizontal dimension of the fluid layer. It is worth noting that the Ra can be interpreted to involve two meanings simultaneously. One is the strength of the baroclinic torque provided by the horizontal temperature difference. The other is the thermal buoyancy generated due to the vertical temperature gradient of the unstable temperature stratification at the destabilizing source, as $T_{1}<T_{\mathrm{i}}<T_{\mathrm{h}}=T_{1}+\Delta \theta$. Both effects are originated from buoyancy; however, we explicitly call the former the baroclinic torque and the latter the destabilizing thermal buoyancy. The presence of the horizontal temperature difference in the thermally equilibrated HC originally produces these two effects, and thus it is inevitable to involve both of the two effects in the Ra for the thermally equilibrated HC. In addition, the contribution of the baroclinic torque alone to the convective motion is considered much smaller than that of the destabilizing thermal buoyancy as evident in Coman et al. [22]; they showed that the circulation strength produced in the thermally equilibrated $\mathrm{HC}$ is $10 \%-20 \%$ of the situation when the same temperature difference is imposed on the vertical direction, i.e., Rayleigh-Bénard convection. Consequently, $\mathrm{Ra}$ is regarded as the primary parameter in the thermally equilibrated $\mathrm{HC}$, and this is quite convenient as it is possible to describe analogy to Rayleigh-Bénard convection.

From the aspects of geophysical and astrophysical interests, transient states of HC developing toward the thermally equilibrated $\mathrm{HC}$ are worth discussing, as the timescale of the development is extremely long. Here we consider the problem setting of the developing stage of HC as illustrated in Fig. 1(b). Both of the temperatures $T_{\mathrm{h}}$ and $T_{1}$ at the top surface are set higher than the interior temperature $T_{\mathrm{i}}$, resulting in $T_{\mathrm{i}}<T_{1}<T_{\mathrm{h}}$. Hence, each side of the top surface acts as a stabilizing 
sources to the internal fluid in a global sense. In this condition, only the temperature difference between $T_{\mathrm{h}}$ and $T_{1}$ generating a baroclinic torque drives a clockwise circulation, as no destabilizing thermal buoyancy is produced. The circulation is then braked by the braking force originated from the restoring force due to the stable temperature gradient across the whole fluid layer. As a result, a circulation localized to the upper region as indicated by black arrows in Fig. 1(b) is formed, unlike the single overturning structures penetrating full height of the fluid layer formed in thermally equilibrated HC illustrated in Fig. 1(a). This kind of flow developments, which can be observed in developing states, is totally different from that in the thermally equilibrated HC. Initial transitions from a quiescent fluid to thermally equilibrated states have been mentioned previously $[4,13,23]$, and it has been reported that those take quite a long time equivalent to or longer than the thermal diffusion timescale due to the absence of a destabilizing source. Similarly, Rossby [2] and Griffiths et al. [24] paid attention to transient evolution of thermally equilibrated HC by providing a sudden temperature change to one of the surfaces imposed a temperature difference leading to a heat flux imbalance. They found that transient adjustments to equilibrium states take a longer time when the temperature of the destabilizing source is weakened, and those can be achieved faster when that of the stabilizing source is weakened. This is because the destabilizing thermal buoyancy is the primary factor to determine the convection structure in thermally equilibrated $\mathrm{HC}$, and the weakening of the destabilizing force sustains only the baroclinic torque while it negates the destabilizing thermal buoyancy. Further, the weakening of the destabilizing force yielded the formation of secondary convection roll due to the friction of the fluid viscosity, as thermal plumes emitted from the weakened destabilizing source cannot penetrate full height of the domain. In such transient evolution toward thermally equilibrated states, it is not possible to describe flow structures by only the Ra defined in Eq. (1) showing the strength of the bulk buoyancy. Instead, the baroclinic torque and the restoring force of the stable temperature stratification become prominent, and these are required to consider as the primary parameters.

Studies of transient processes of developing HC appeared less frequently. Even in the studies, thermal buoyancy destabilizing fluid layer has been always treated as an important parameter. For instance, a balanced situation of convection driven by both baroclinic torque and thermal buoyancy was investigated experimentally by $\mathrm{Lu}$ et al. [8,9] and numerically by Niino et al. [11] from the perspective of the temporal evolution problem for urban heat-island circulation. These analogs of heat-island circulation suggest regime transitions occur due to the balancing of baroclinic torque and thermal buoyancy. Some previous studies [4,13,23] are only a few cases dealing with the the developing HC, i.e., the initial transitions without destabilizing source from one thermally equilibrated $\mathrm{HC}$ to the other thermally equilibrated HC. However, they did not describe details of flow developments in the transitions, as they focused on thermally equilibrated states at which the destabilizing thermal buoyancy is dominant. Flow structures in developing $\mathrm{HC}$ without destabilizing source has been addressed only in Mori and Niino [10] from their theoretical and numerical approaches. In their work, linear stable temperature stratification was considered for base states, and heat removal was imposed at a part of the bottom. The formulation of the problem is given as a two-dimensional vorticity equation,

$$
\frac{\partial \eta}{\partial t}+\frac{\partial \eta}{\partial x} \frac{\partial \psi}{\partial z}-\frac{\partial \eta}{\partial z} \frac{\partial \psi}{\partial x}=g \beta \frac{\partial T}{\partial x}+v \nabla^{2} \eta
$$

and a thermodynamic equation,

$$
\frac{\partial T}{\partial t}+\frac{\partial T}{\partial x} \frac{\partial \psi}{\partial z}-\frac{\partial T}{\partial z} \frac{\partial \psi}{\partial x}=\gamma \frac{\partial \psi}{\partial x}+\kappa \nabla^{2} T
$$

where $\psi, \eta, T$, and $\gamma$ are, respectively, the stream function, $y$-component of vorticity, temperature, and the vertical temperature gradient of the base temperature field, $T(z)=T_{0}+\gamma z$. Under this condition, the first term on the right-hand side of Eq. (2) is the baroclinic vorticity production term, which is the main source driving the convective motion. The first term on the right-hand side of Eq. (3) is the restoring term of stable temperature stratification, which stabilizes the convective 
motion. With these formulations explicitly denoting the effect of base stable stratification as $\gamma$, Mori and Niino [10] suggested three flow regimes based on the time and degree of the stratification, namely, diffusion, gravity current, and gravity wave regimes, and found self-similar solutions for each regime in laterally infinite domains. These flow regimes are totally different from those in thermally equilibrated states (see, e.g., $[1,13]$ ). To incorporate the baroclinicity and the restoring force of the stable temperature stratification, Mori and Niino [10] introduced the nondimensional stratification parameter $\gamma^{\prime}$. It is a ratio of temperature gradients arising from the restoring force of the stable temperature stratification $\gamma$ and the baroclinic vorticity $\xi$ denoted as

$$
\gamma^{\prime}=\frac{\gamma}{\xi}=\gamma \frac{\left(\frac{\kappa^{2}}{g \beta \Delta \theta}\right)^{1 / 3}}{\Delta \theta}=g \beta \gamma\left(\frac{\sqrt{\kappa}}{g \beta \Delta \theta}\right)^{4 / 3} .
$$

It is difficult to define the length scale for the baroclinic torque, however, Mori and Niino [10] employed $\left[\kappa^{2} /(g \beta \Delta \theta)\right]^{1 / 3}$ for it. This length represents the distance that the heated fluids at the stabilizing source can possess sufficient thermal buoyancy compared to the surroundings even with losing their buoyancy by thermal diffusion, i.e., the length that the baroclinic torque can effectively act against the thermal diffusion. The importance of the work of Mori and Niino [10] is the explicit consideration of the restoring force due to the stable temperature stratification. The baroclinicity driving the convective motion and restoring force of the stable temperature stratification are taken into account by defining $\gamma^{\prime}$ as the primary factor in the developing HC. In contrast, $\gamma^{\prime}$ is not exposed in thermally equilibrated $\mathrm{HC}$ as $\gamma$ becomes zero.

Although Mori and Niino [10] showed the importance of $\gamma^{\prime}$ in the developing HC, there are two points expected to be addressed to fully understand the physics in developing HC. One is the influence of side walls; only a laterally infinite domain was investigated, while thermally equilibrated $\mathrm{HC}$ have been examined in finite domains. It is obvious that the self-similar solutions found by Mori and Niino [10] cannot be held in finite domains due to its geometric restriction. The contribution of $\gamma^{\prime}$ to the developing $\mathrm{HC}$ in a finite domain is worth discussing from the perspective of transient adjustment processes of thermally equilibrated HC [2,24], since the developing HC finally reaches the thermally equilibrated $\mathrm{HC}$ after a sufficiently long time due to thermal diffusion. Another is that $\gamma^{\prime}$ was considered as a given parameter irrespective of flow structures. As the convective motion transports heat from the horizontal surface, the degree of stable temperature stratification should be modified with time. In particular, this issue becomes prominent for considering a finite domain. These issues should be addressed to understand developing $\mathrm{HC}$ as a transient process arising in thermally equilibrated $\mathrm{HC}$.

In the present study, we investigate developing $\mathrm{HC}$ at which no destabilizing source exists, through experimental studies. Using a rectangular container as the fluid layer, convective flow structures developed in a finite domain are clarified. Further, we focus on the effect of time relaxation of the stable temperature stratification on flow structures of the developing HC. These conditions are realized in laboratory experiments by imposing differential heating at a top of a cold fluid confined within thermally insulated walls. Transient processes developing toward thermally equilibrated HC are quantified by adopting optical visualization techniques. Section II presents the experimental setup, procedures and a series of data analyses for acquiring flow characteristics. Temporal evolution of flow structures and the effect of stable stratification on $\mathrm{HC}$ are explained in Sec. III and discussed in detail in Sec. IV. We summarize the findings of the present study in Sec. V.

\section{EXPERIMENT}

\section{A. Experimental setup}

A rectangular container with internal dimensions of $200 \mathrm{~mm}(4 L) \times 50 \mathrm{~mm}(L) \times 50 \mathrm{~mm}(L)$ in $x, y$, and $z$ directions was used for the experimental investigations. The fluid container is illustrated in Fig. 2. The sides and bottom of the container consisted of acrylic walls thicker than $10 \mathrm{~mm}$. The top of the fluid layer was closed by four copper blocks, with each block having an internal cavity 


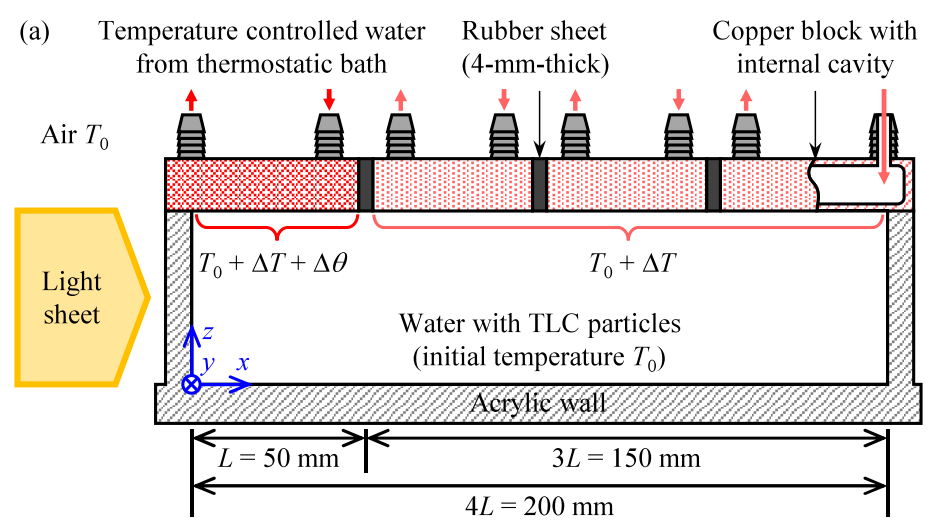

(b)

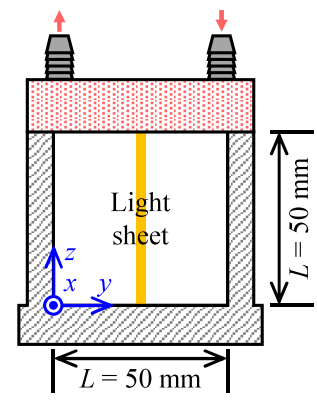

FIG. 2. Schematic of the rectangular fluid container: (a) front view and (b) side view.

allowing the circulation of water from thermostatic baths. The copper blocks were aligned and isolated by 4-mm-thick rubber sheets to prevent heat exchange between adjacent blocks and allow the control of the temperatures of the blocks individually. Degassed distilled water (with the Prandtl number of $\operatorname{Pr}=v / \kappa \sim 7$ at $T=20^{\circ} \mathrm{C}$ ) was used as the test fluid, and two types of thermochromic liquid crystal (TLC) particles (having a mean diameter $\sim 15 \mu \mathrm{m}$, mean density $1.02 \times 10^{-3} \mathrm{~kg} / \mathrm{m}^{3}$; KWN-2025 and KWN-2530, Japan Capsular Products Inc.) were seeded for flow visualization. The approximate temperature range was $5 \mathrm{~K}$ for the two types of TLC particles; one type of TLC particle showed coloration at $20-25^{\circ} \mathrm{C}$ and the other showed coloration at $25-30^{\circ} \mathrm{C}$. Accordingly, two color cycles of the TLC particles were expected to be observed from 20 to $30^{\circ} \mathrm{C}$. The thermal conductivity of water, $\sim 0.6 \mathrm{~W} /(\mathrm{m} \mathrm{K})$, is three times larger than that of acrylic, $\sim 0.2 \mathrm{~W} /(\mathrm{m} \mathrm{K})$. The thermal condition at the side and bottom walls thus cannot be considered fully adiabatic, and there is a relatively small temperature difference between the bulk of the fluid and the ambient temperature. In the preliminary tests, however, we confirmed that the flows on the $y-z$ plane were negligibly slow, and therefore, the flow fields explored in the present study can be regarded as (quasi-) two-dimensional as expected in the work by Mori and Niino [10]. The vertical cross section at $y=0.5 \mathrm{~L}$ was visualized using a white light sheet generated by a cylindrical lens and a halogen lamp for quantitative measurements. With these optical configurations, the TLC particles allowed the visualization of temperature fields as variations in color as red, green, and blue for lower to higher temperature in the specific ranges. In addition, particle motions can be traced adopting well-known techniques such as particle image velocimetry (PIV) and particle-tracking velocimetry (PTV), and flow fields can be measured as detailed in Sec. II C. The use of the TLC particles for the purpose of PIV was introduced in our earlier works [25,26]. A CMOS color camera (DFK33UP5000, Imaging Source Co.) was set in front of the fluid container to record particle motions in the illuminated $x-z$ plane at $y=0.5 L$. The image resolution was 2400 pixel $\times 600$ pixel to $200 \mathrm{~mm}(4 L) \times 50 \mathrm{~mm}(L)$. For direct measurements of the temperature of the top surface on which a horizontal temperature difference was imposed, seven horizontally aligned thermistor sensors having a diameter of $1.5 \mathrm{~mm}$ were inserted into the fluid layer and fixed close to the top surface at $z \sim 0.98 L$. The thermistor sensors measured the temperature with accuracy better than $\pm 0.1 \mathrm{~K}$ and precision of less than $\pm 0.02 \mathrm{~K}$. The details of temperature measurements are described in Sec. II C.

\section{B. Experimental procedure}

To systematically investigate the flow structures observed in developing HC through laboratory experiments, we set two variable experimental parameters, namely, the vertical temperature difference $\Delta T$ and horizontal temperature difference $\Delta \theta$. Experimental measurements were made in a room at a controlled temperature of $T_{0}=20^{\circ} \mathrm{C}$. Initially, the fluid container was filled with a 
quiescent fluid at a set temperature $T_{0}$, and temperatures of the four copper blocks at the top of the fluid layer were maintained also at $T_{0}$. At $t=0$, the set temperature of one of the thermostatic baths connected to the left-end copper block was increased to $T=T_{0}+\Delta T+\Delta \theta$ and that of the other thermostatic bath connected to the right three copper blocks was increased to $T_{0}+\Delta T$. Here $\Delta T$ is a positive temperature difference imposed at the three right blocks relative to the initial temperature $T_{0}$ to generate stable temperature stratification at $L \lesssim x \leqslant 4 L . \Delta \theta$ is a positive temperature difference imposed at the left-end copper block relative to the three right copper blocks to realize stable temperature stratification at $0 \leqslant x \lesssim L$. Note that the thermostats typically required a few minutes to increase the temperature to the set values, and the initial transient flows are thus excluded from the following discussions. For each condition, continuous image acquisitions were made at a frequency of $10 \mathrm{~Hz}$ for the first $30 \mathrm{~min}$ and for $1 \mathrm{~min}$ at every $30 \mathrm{~min}$ from the initiation of the heating up to $3 \mathrm{~h}$, corresponding to 0.6 in the thermal diffusion timescale $\tau_{\kappa}=L^{2} / \kappa$.

With the explicitly defined temperature differences, $\Delta \theta$ and $\Delta T$, we calculate the nondimensional stratification parameter $\gamma^{\prime}$, denoting the balance of the braking force arising from the restoring force of the stable temperature stratification and the baroclinic torque originated from the horizontal temperature gradient. It is convenient if the stratification parameter is given a priori as fixed values, and we thus consider here the linearly stratified condition with a mean temperature gradient of

$$
\gamma_{\text {lin }}=\frac{T_{\text {wall }}-T_{0}}{L}
$$

where $T_{\text {wall }}$ is the mean temperature of the heating copper blocks simply given as

$$
T_{\text {wall }}=\frac{1}{4}\left(T_{0}+\Delta T+\Delta \theta\right)+\frac{3}{4}\left(T_{0}+\Delta T\right) .
$$

Accordingly, the mean vertical temperature gradient is expressed as

$$
\gamma_{\text {lin }}=\frac{4 \Delta T+\Delta \theta}{4 L}
$$

With these notations, the nondimensional stratification parameter in the linearly stratified case $\gamma_{\text {lin }}^{\prime}$ is expressed as

$$
\gamma_{\text {lin }}^{\prime}=\frac{\gamma_{\text {lin }}}{\xi}=\frac{g \beta(4 \Delta T+\Delta \theta)}{4 L}\left(\frac{\sqrt{\kappa}}{g \beta \Delta \theta}\right)^{4 / 3} .
$$

For reference, conventionally used $\mathrm{Ra}$ is also calculated from Eq. (1) using $W=4 L$ as the horizontal length scale. Please note that Ra corresponds to the maximum buoyancy potentially existing in the system under thermally equilibrated states. Thus, Ra calculated here is the equivalent Rayleigh number realized when the same horizontal temperature difference is imposed in thermally equilibrated HC.

In the present study, the imposed temperature differences were chosen within feasible ranges in the laboratory environments with yielding convective motions. For both $\Delta \theta$ and $\Delta T$, four different temperature differences were imposed resulting in 16 parameters, and the explored parameters are summarized in Table I and plotted in Fig. 3. Figure 3(a) shows plots in the domain of experimentally controllable parameters $(\Delta \theta-\Delta T$ with a unit of $\mathrm{K})$, while plots in the domain of nondimensional parameters $\left(\mathrm{Ra}-\gamma_{\text {lin }}^{\prime}\right)$ are shown in Fig. 3(b). The color contours in Fig. 3(a) represent the nondimensional stratification parameter $\gamma_{\text {lin }}^{\prime}$. According to Mori and Niino [10], flow regimes in a laterally infinite system can be categorized into three regimes: diffusion, gravity current, and gravity wave regimes depending on $\gamma_{\text {lin }}^{\prime}$ and time from heating. The diffusion regime may not be observed in the experiments, because the transition from the regime will be completed on a timescale $O\left(<10^{0} \mathrm{~s}\right)$ in cases that water is used as the test fluid. In the present study, the initial temperature of the fluid is $T_{0}$ at $t=0$, and the dimensional stratification parameter $\gamma$ thus asymptotes from $\infty$ to 0 with the growth of thermal boundary layers at the top, which defines the length scale of the stratification. Most of the presently explored parameters $\gamma_{\text {lin }}^{\prime}=O\left(10^{-4}-10^{-1}\right)$ are expected to be in the gravity current regime after a sufficiently long time for thermal diffusion according to the 
TABLE I. Experimental parameters explored in the present study. For all conditions, the initial fluid temperature was $T_{0}=20^{\circ} \mathrm{C}$.

\begin{tabular}{lcccc}
\hline \hline Case & $\Delta \theta[\mathrm{K}]$ & $\mathrm{Ra}$ & $\Delta T[\mathrm{~K}]$ & $\gamma_{\text {lin }}^{\prime}$ \\
\hline 1 & 1 & $1.1 \times 10^{8}$ & 1 & $5.4 \times 10^{-3}$ \\
2 & & 2 & $9.6 \times 10^{-3}$ \\
3 & & 5 & $2.3 \times 10^{-2}$ \\
4 & 2 & 10 & $4.4 \times 10^{-2}$ \\
5 & $2.3 \times 10^{8}$ & 1 & $2.6 \times 10^{-3}$ \\
6 & & 2 & $4.3 \times 10^{-3}$ \\
7 & & 5 & $9.4 \times 10^{-3}$ \\
8 & 5 & 10 & $1.8 \times 10^{-2}$ \\
9 & & 1 & $1.1 \times 10^{-3}$ \\
10 & & 2 & $1.6 \times 10^{-3}$ \\
11 & & 5 & $3.1 \times 10^{-3}$ \\
12 & & 10 & $5.6 \times 10^{-3}$ \\
13 & & 1 & $7.0 \times 10^{-4}$ \\
14 & & 2 & $9.0 \times 10^{-4}$ \\
15 & & 5 & $1.5 \times 10^{-3}$ \\
16 & & & 10 & $2.5 \times 10^{-3}$ \\
\hline \hline
\end{tabular}

condition $\gamma_{\text {lin }}^{\prime} \ll 1$, if the system is horizontally infinite. In the present study, we use a rectangular container for the fluid layer, and the horizontal length scale is regulated up to $4 L$. This geometric constraint should affect the flow regimes observed in the present study. Suppose the same $\Delta \theta$ is imposed in thermally equilibrated $\mathrm{HC}$, the resultant Ra ranges $10^{8}-10^{9}$. These parameters are sufficiently strong to form a single overturning convection cell penetrating the full depth of the domain in a thermally equilibrated state.

\section{Data acquisition methodology}

In Fig. 4 an example of flow visualization using the two types of TLC particles with the conditions $\Delta T=2 \mathrm{~K}$ and $\Delta \theta=5 \mathrm{~K}$ taken at $t=30 \mathrm{~min}$ is shown as a color image. Colors of
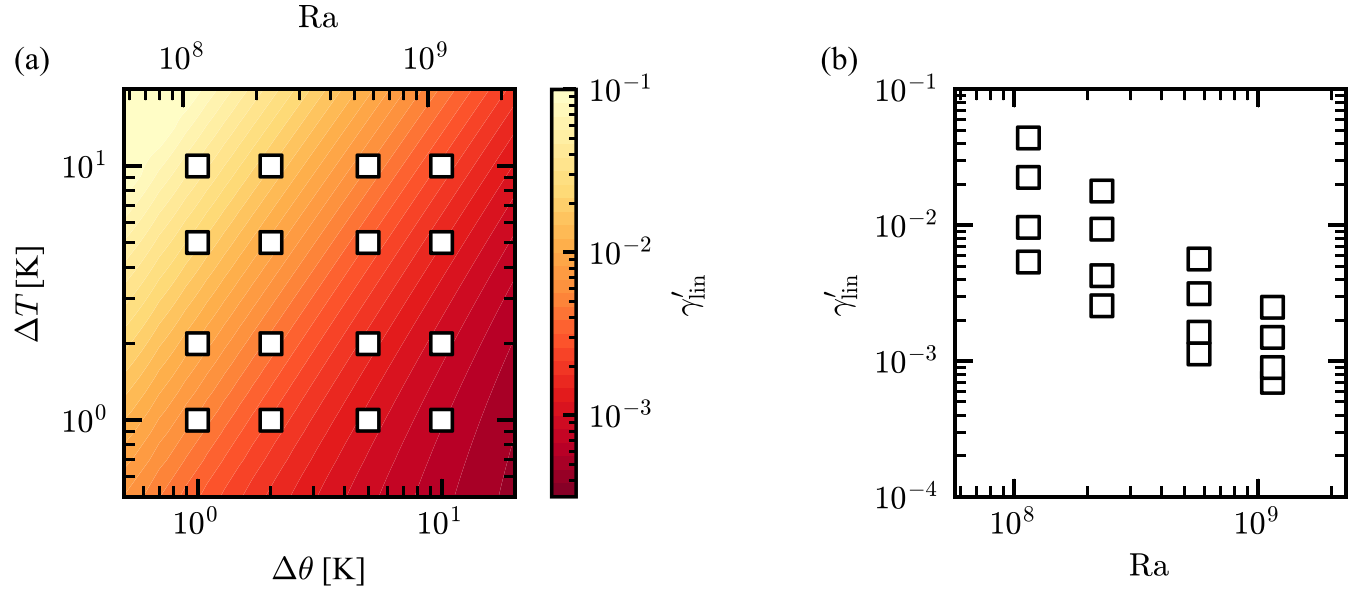

FIG. 3. Parameters explored in the present study: (a) plots of experimentally controllable parameters, horizontal temperature difference $\Delta \theta$, and vertical temperature difference $\Delta T$ and (b) plots of nondimensional parameters in the $\mathrm{Ra}-\gamma_{\text {lin }}^{\prime}$ domain. Color contours in (a) represent $\gamma_{\text {lin }}^{\prime}$ values. 


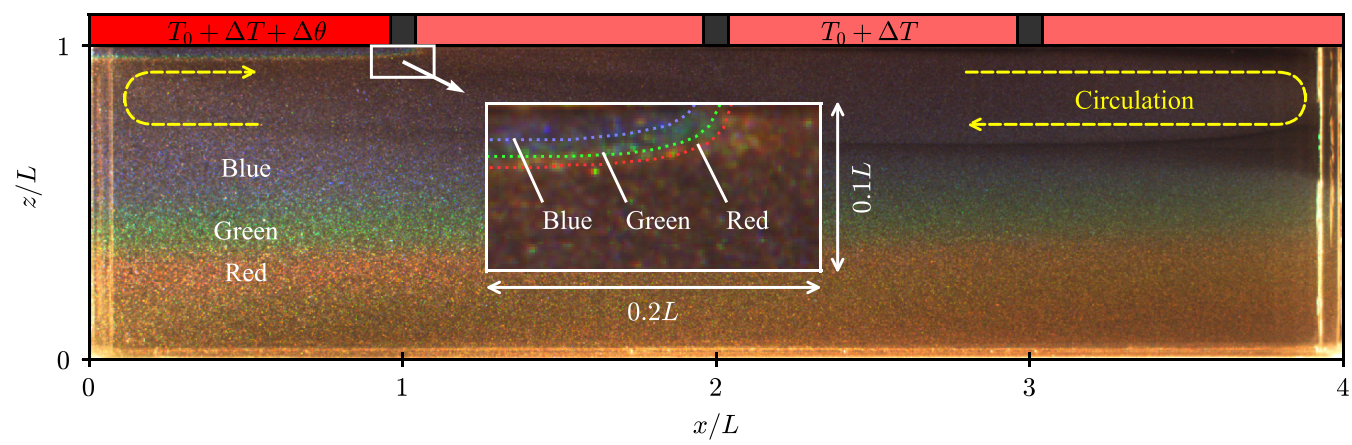

FIG. 4. Example of flow visualization using the TLC particles of two different temperature ranges at $t=30 \mathrm{~min}$ with the condition of $\Delta T=2 \mathrm{~K}$ and $\Delta \theta=5 \mathrm{~K}$. Red and black panels above the fluid layer indicate the locations of the heating copper blocks and insulating rubber sheets. A magnified view of the region enclosed by the solid square of $0.2 L \times 0.1 L$ is presented in the inset, and qualitative isotherms are indicated by dotted lines. Colors of the TLC particles change as red, green, and blue according to the increase in fluid temperature. The color contrast is adjusted to enhance visibility.

the TLC particles can be qualitatively understood as the isotherms. The first color cycle of one type of TLC particle can be seen as red, green, and blue regions from the bottom to top of the fluid layer, and this gradual color change corresponds to an increase in temperature, showing stable temperature stratification. Note that the color change of the TLC particle is not linear, and the temperature range from red to green is narrow $<1 \mathrm{~K}$ out of $5 \mathrm{~K}$. The second color cycle of the other type of TLC particle appears within a shallow region immediately beneath the left-end copper block $(0 \leqslant x \lesssim L)$ meaning a steep temperature gradient beneath the block; a magnified view of this second cycle in the region enclosed by the solid square $(0.2 L \times 0.1 L)$ is shown in the inset. The colors blue, green, and red appear in order from upper left to lower right. Considering the color changes in the magnified region, isotherms are qualitatively drawn as the dotted lines. Nearly vertical isotherms are identified at $x \sim L$ and may produce baroclinic torque. Overall, except for the magnified region, the test fluid in the rectangular container is found to form stable temperature stratification with different degrees of stratification along the horizontal direction. With stable temperature stratification, a clockwise circulating motion forms beneath the top copper blocks owing to the baroclinic torque as indicated by the yellow arrows. This circulation is measured quantitatively adopting image processing techniques in the following.

In advance of the quantification of flow fields, the source of horizontal circulation is examined using the measurement of temperature. To confirm the vertically aligned isotherms for generating baroclinic torque qualitatively illustrated in the magnified inset of Fig. 4, horizontal temperature profiles beneath the copper blocks are directly measured using the thermistor sensors. Measurement points of the seven sensors were set immediately beneath the copper blocks at $z \sim 0.98 L$ and $x=$ $L, L \pm 0.1 L, L \pm 0.2 L$, and $L \pm 0.5 L$, which cover the region around $x=L$ where the horizontal temperature difference $\Delta \theta$ was imposed. An example of temperature measurements as a function of the $x$ position for $\Delta T=5 \mathrm{~K}$ and $\Delta \theta=5 \mathrm{~K}$ is shown in Fig. 5. For clarity, temperature profiles measured every $20 \mathrm{~min}$ are plotted. The squares show the temperatures measured by the thermistor sensors and are colored according to the time elapsed from the initiation of heating. The horizontal dotted lines are the set temperatures of $T=T_{0}, T_{0}+\Delta T$, and $T_{0}+\Delta T+\Delta \theta$ from bottom to top. At $t=0 \mathrm{~min}$, the temperatures of all sensors record $T \simeq T_{0}$ because the initial temperature of the test fluid is $T_{0}$. At $t \geqslant 20 \mathrm{~min}$, the measured temperatures increase, and they do not change with time until $t=180 \mathrm{~min}$. Since the temperatures at the top surfaces were kept constantly, the measured temperatures at different time are fully overlying in Fig. 5(a) and hard to be distinguished. The measured temperatures are slightly different from the set temperature of the copper blocks as the sensors measure the fluid temperature at $z \sim 0.98 \mathrm{~L}$. These horizontal temperature profiles measured 


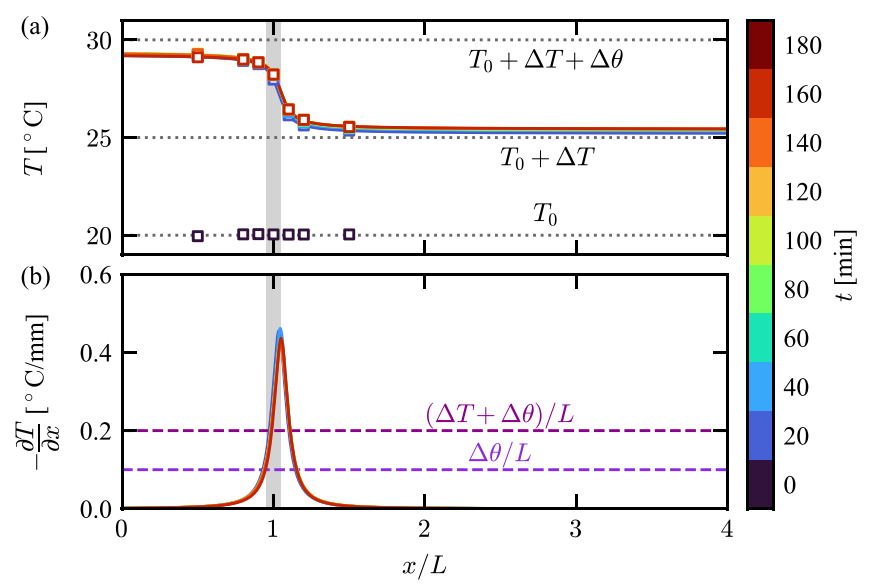

FIG. 5. Example of temperature measurements made beneath the copper blocks at $z \sim 0.98 L$ under the conditions $\Delta T=5 \mathrm{~K}$ and $\Delta \theta=5 \mathrm{~K}$ : (a) temperatures measured by the thermistor sensors (squares) and corresponding fitting curves and (b) horizontal temperature gradients derived from the fitting curves. Each plot and curve is colored according to the time elapsed from the initiation of heating. Horizontal dotted lines in (a) show the set temperatures of $T=T_{0}, T_{0}+\Delta T$, and $T_{0}+\Delta T+\Delta \theta$ from bottom to top. Horizontal dashed lines indicate typical temperature gradients obtained using the set constants. Regions shaded gray show horizontal positions of the insulating rubber sheet, at which the horizontal temperature difference $\Delta \theta$ is imposed.

by the sensors can be expressed as

$$
T(x)=\frac{A}{\pi} \tan ^{-1}\left[B\left(x-x_{c}\right)\right]+C,
$$

where $A, B$, and $C$ are constants obtained by least-squares fitting. Additionally, $x_{c}$ denotes the $x$ position at which the maximum horizontal temperature gradient is imposed. The fitting curves are drawn as solid lines colored by the elapsed time in Fig. 5(a). Using the fitting curve in Eq. (9), the horizontal temperature gradient $\partial T / \partial x$ is derived as

$$
\frac{\partial T(x)}{\partial x}=\frac{A B}{\pi\left\{\left[B\left(x-x_{c}\right)\right]^{2}+1\right\}} .
$$

In Fig. 5(b) the absolute values of horizontal temperature gradients at each moment are drawn as solid lines colored according to the elapsed time. For reference, typical temperature gradients obtained using characteristic temperature differences and lengths are drawn as horizontal dashed lines. One peak in $-\partial T / \partial x$ stands slightly downstream from $x=L$ for each moment after $t=$ 20 min owing to heat transport from the left-end copper block toward the downstream, and the shape of the curves hardly changes with time. In the vorticity equation given as Eq. (2), the horizontal temperature gradient corresponds to the baroclinic vorticity production term. Accordingly, the peak in Fig. 5(b) is the source of the circulating motions localized in the upper region of the fluid layer, and the driving force in the system is thus considered always constant once the temperatures of the copper blocks are fixed. Meanwhile, the intensity of the stratification may relax with time owing to the thermal insulating condition at the side and bottom walls, and the restoring force of stable stratification thus weakens with time. The circulating motions indicated in Fig. 4 therefore show temporal developments, which will be detailed in Sec. III.

The circulating motions illustrated qualitatively in Fig. 4 can be quantified through particle image analyses like PIV for the visualized images containing the TLC particles. In most cases investigated in the present study, the circulating motions are confined in the narrow upper region of the fluid layer as shown in Fig. 4, and there may be large fluid deformation at the rotational axis 

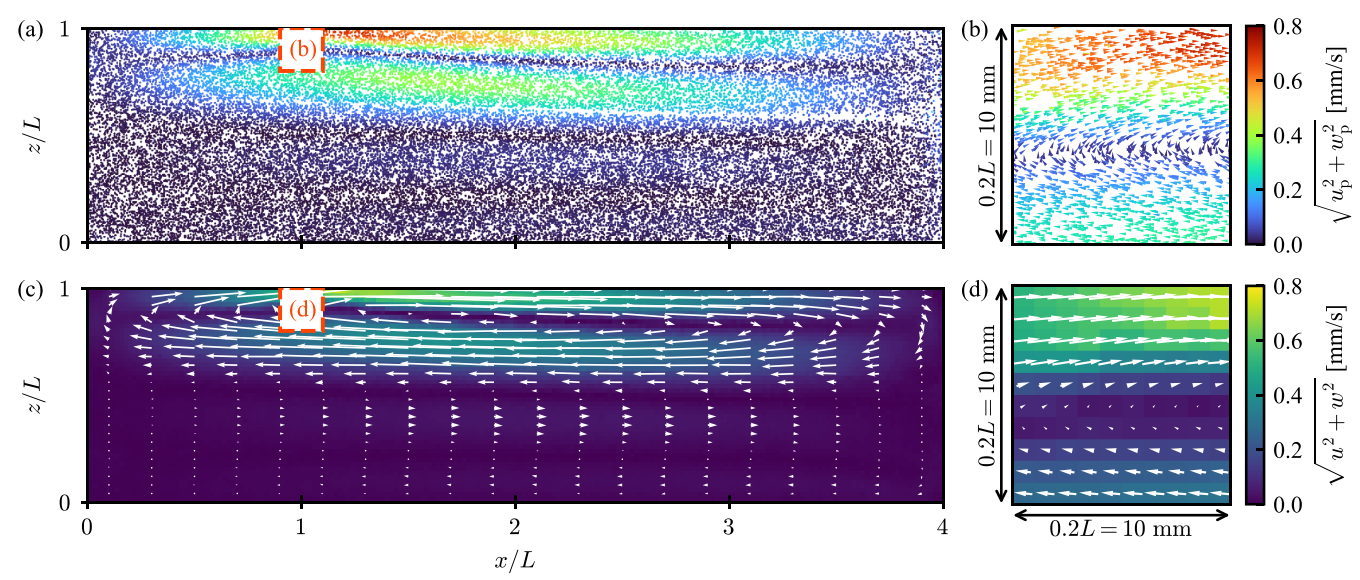

FIG. 6. Example of velocity field measurements at $t=90 \mathrm{~min}$ under the conditions of $\Delta T=2 \mathrm{~K}$ and $\Delta \theta=5 \mathrm{~K}$ : (a) particles tracked through PTV analysis indicated by dots colored according to speed; (b) magnified view of the particle vectors in the region enclosed by the dashed square in (a); (c) overview of the interpolated velocity fields; and (d) magnified view. Only $1 \%$ and $4 \%$ of the tracked particles are displayed in (a) and (b), respectively. Interpolated velocity vectors are superposed on the color contours of speeds as white arrows in (c) and (d), and only one out of 10 and one out of two vectors are plotted in the $x$ and $z$ directions, respectively, for the sake of clarity in (c).

of roll structures. Thus, velocity field measurements made adopting standard PIV algorithms, direct cross-correlation, and fast Fourier transform (FFT) correlation methods may not be appropriate owing to the requirement of interrogation windows. The image resolution of the present optical setting is better than $0.1 \mathrm{~mm} /$ pixel (i.e., 12 pixels for $1 \mathrm{~mm}$ ), and it is possible to identify each TLC particle as the particle image spreads across two or three pixels on the visualized image after background subtraction. We therefore adopt PTV analysis based on the nearest-neighbor method to measure velocity fields. An example of tracked particles is shown in Fig. 6(a) as dots colored according to speed. More than 40 million particles were tracked in total during $1 \mathrm{~min}$ in the case presented in Fig. 6(a), where only $1 \%$ are plotted as dots. Particle velocity vectors in the region enclosed by the dashed square in Fig. 6(a) are shown in Fig. 6(b) as arrows colored according to speed. Only $4 \%$ of the tracked vectors are shown for clarity in Fig. 6(b). The TLC particles are homogeneously distributed in space, and dense particle vectors are thus obtained. A high-speed region is localized immediately beneath the copper blocks imposed with a horizontal temperature difference $\Delta \theta$ [Fig. 6(a)], and a clockwise circulating motion is recognized [Fig. 6(b)].

To assist the series of analyses conducted afterward, velocity vectors $\boldsymbol{u}=[u, w]$ on regular grids, which have a resolution of $1 \mathrm{~mm}$ in $x$ and $z$ directions, are calculated using all particle vectors $\boldsymbol{u}_{\mathrm{p}}=\left[u_{\mathrm{p}}, w_{\mathrm{p}}\right]$ measured during a period of $1 \mathrm{~min}$, as the flow fields hardly change during this short time. We employ Shepard's method [27] for the grid interpolation, and the squares of the inverse distances between the regular cells and the particle positions are used as the weight for the interpolation. That is, $u$ and $w$ are calculated as

$$
u=\sum_{i}\left|\boldsymbol{r}_{i}\right|^{-2} u_{\mathrm{p} i} / \sum_{i}\left|\boldsymbol{r}_{i}\right|^{-2}, \quad w=\sum_{i}\left|\boldsymbol{r}_{i}\right|^{-2} w_{\mathrm{p} i} / \sum_{i}\left|\boldsymbol{r}_{i}\right|^{-2},
$$

where $\boldsymbol{r}_{i}=\boldsymbol{x}-\boldsymbol{x}_{\mathrm{p} i}$ is the Euclidean distance between the cells $\boldsymbol{x}$ and the particle positions $\boldsymbol{x}_{\mathrm{p} i}$. As all particle vectors measured during $1 \mathrm{~min}$ are used in the interpolation, the interpolated velocity $\boldsymbol{u}$ gives the temporal averaged velocity, while the flow field hardly changes. An example of the interpolated velocity field is shown in Fig. 6(c), and a magnified view of the region enclosed by the dashed square in Fig. 6(c) is shown in Fig. 6(d). Interpolated vectors represented by white arrows 

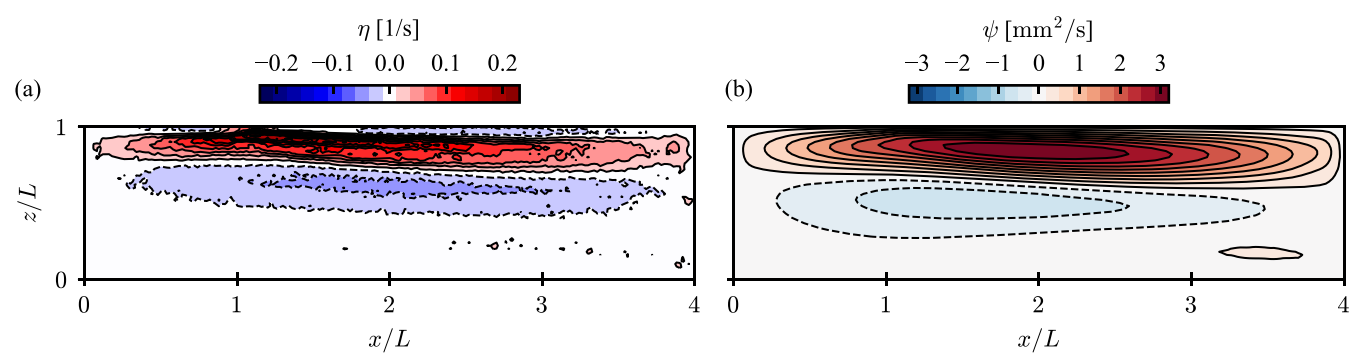

FIG. 7. Example of vorticity and stream function fields at $t=150 \mathrm{~min}$ under the conditions $\Delta T=2 \mathrm{~K}$ and $\Delta \theta=5 \mathrm{~K}$ : (a) vorticity $\eta$ field and (b) stream function $\psi$ field. Positive and negative contours are drawn using solid and dashed lines.

are superposed on the color contours of the speeds in Figs. 6(c) and 6(d). Only one out of 10 and one out of two vectors are plotted in the $x$ and $z$ directions, respectively, for the sake of clarity, in Fig. 6(c). The horizontal flows driven in the region where a horizontal temperature difference is imposed impinge on the sidewall at the right end and turn toward the $-z$ direction. The turned flow penetrates the quiescent fluid layer up to certain depths and turns again toward the $-x$ direction to generate circulating motion. The clockwise circulation occupying the upper half of the fluid layer is well reconstructed in the interpolated velocity fields.

To directly compare the governing equations given as Eq. (2), the out-of-plane vorticity $\eta$ and the stream function $\psi$ are calculated from the interpolated velocity vector fields $\boldsymbol{u}(x, y)$. Here the out-of-plane vorticity $\eta$ is expressed as

$$
\eta=\frac{\partial u}{\partial z}-\frac{\partial w}{\partial x}
$$

and the stream function $\psi$ is expressed as

$$
\nabla^{2} \psi=\eta
$$

The vorticity distributions are calculated using the second-order central-difference scheme for the interpolated velocity fields according to Eq. (12). The stream function distribution can be computed by solving the Poisson equation [Eq. (13)] adopting the successive over-relaxation (SOR) method [28] with the boundary conditions of $\psi=0$ at the solid walls. Examples of the vorticity and stream function fields are shown in Fig. 7. In Fig. 7(a) the vorticity field at $t=150$ min under the conditions $\Delta T=2 \mathrm{~K}$ and $\Delta \theta=5 \mathrm{~K}$ is shown as color contours, while the corresponding stream function field is shown as color contours in Fig. 7(b). Positive and negative contours are respectively drawn using solid and dashed lines, and the contour lines in Fig. 7(b) coincide with the streamlines of the flow. The order of $\psi$, a few to 10 times larger than the thermal diffusivity $\kappa$, is consistent with the results of previous work [5]. The clockwise circulation beneath the top copper blocks is now visible both in vorticity and stream function fields as large positive regions in the upper half of the fluid layer. In addition, the second negative and third positive roll structures become visible beneath the first roll structure in Fig. 7(b). Compared with the relatively noisy vorticity distributions in Fig. 7(a), the stream function field is smooth as the iterations of the SOR method provide smooth stream function distributions.

The depths of the circulating flow, that is the thickness of the roll structures $\delta_{\text {roll }}$, can be measured using the stream function fields, as the lower boundary of the roll is clearer in Fig. 7(b) than in Fig. 7(a). We here define roll regions as coherent regions having more than $5 \%$ intensity of the maximum stream function value $\psi_{\max }$ throughout the domain. The bottom of the roll structures are slightly inclined to the lower right as shown in Fig. 7(b). This inclined roll boundary is considered to originate from the horizontally differing degree of stable stratification $\gamma$; the left side has strong and the right side has weak stable stratification as the imposed heating is higher on the left side. 


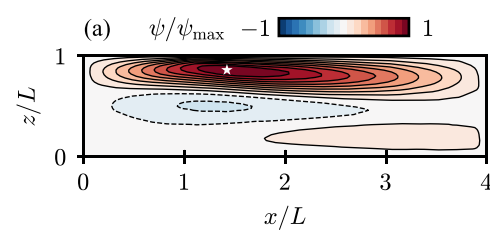

(b) $\psi / \psi_{\max }$

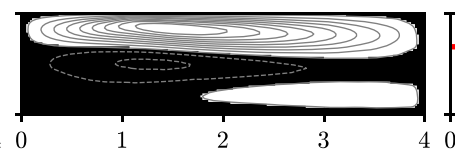

$x / L$ (c)

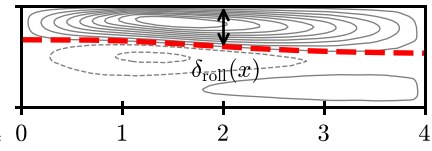

FIG. 8. Extraction of the roll region according to the intensity of the stream function field at $t=180 \mathrm{~min}$ under the conditions $\Delta T=2 \mathrm{~K}$ and $\Delta \theta=2 \mathrm{~K}$ : (a) stream function field normalized by the maximum value $\psi_{\max }$; (b) binarized stream function field with a threshold at $5 \%$ intensity of the maximum value; and (c) bottom line of the roll structure fitted by the Gompertz function, showing thickness of the roll structure $\delta_{\text {roll }}(x)$. The position of $\psi=\psi_{\max }$ is indicated by a white star in (a). Contour lines of $\psi / \psi_{\max }$ are superposed on (b) and (c) as gray solid (positive) and dashed (negative) lines.

This trend is identical to all the explored parameters. The roll thickness at any horizontal position $\delta_{\text {roll }}(x)$ is then fitted by an asymmetric function, the Gompertz function, in the form

$$
\delta_{\text {roll }}(x)=A \exp [-B \exp (-C x)]+D,
$$

where $A, B, C$, and $D$ are constants obtained by least-squares fitting, with $B$ and $C$ being positive. From this fitting, the roll region is identified as the closed region of $0 \leqslant x \leqslant 4 L$ and $L-\delta_{\text {roll }}(x) \leqslant$ $z \leqslant L$. A series of analyses for computing the roll thickness $\delta_{\text {roll }}$ is presented in Fig. 8. The original stream function field is shown in Fig. 8(a), and the position at which $\psi=\psi_{\max }$ is indicated by a white star. The position is located downstream from $x=L$, and its horizontal ordinate roughly coincides with that at which $u=u_{\max }$ as shown in Fig. 6 . The stream function field is then binarized with a threshold of 5\% intensity of $\psi_{\max }$. Regions exceeding the threshold are shown as white in Fig. 8(b). From the binarized stream function field shown in Fig. 8(b), the level corresponding to the bottom of the extracted roll region immediately beneath the top surface is fitted using Eq. (14), and the fitted curve is drawn as a red dashed line in Fig. 8(c).

\section{RESULTS}

\section{A. Temporal evolution of horizontal convection}

During the transient process toward thermally equilibrated state, the convection structure formed beneath the heating blocks develops with time. To investigate details of the flow structures of developing $\mathrm{HC}$, the temporal evolution of the flow fields under fixed conditions $\Delta T=2 \mathrm{~K}$ and $\Delta \theta=2 \mathrm{~K}$ is shown in Fig. 9. Figures 9(a)-9(h) shows the overall view of the stream function fields (color contours) and the lower ends of the roll structures corresponding to the roll thicknesses (red dashed lines), at the moments noted above each subfigure. Each color contour is scaled by the common color bar, which is roughly adjusted to the maximum value at $t=180 \mathrm{~min}$ [Fig. 9(h)] and shown above the stream function fields. At $t=10 \mathrm{~min}$ as shown in Fig. 9(a), the stream function field is weak; however, the roll structure in the upper region already extends horizontally to $\sim 4 L$, the width of the fluid container, with very small thickness $\sim 0.3 \mathrm{~L}$. With time, the stream function within the upper roll region increases, representing the strengthening of the roll structure. Additionally, the roll region increases with the strengthening of the roll structure, and the roll thickness increases as the roll structure is able to grow only in the $-z$ direction owing to the horizontal confinement.

Adopting the data acquisition methods described in Sec. IIC, various flow characteristics of horizontal convection can be quantified. In Fig. 9(i) changes in the maximum stream function $\psi_{\max }$ with time are plotted as circles, and changes in the roll thickness $\delta_{\text {roll }}$ are shown. $\delta_{\text {roll }}$ changes with the $x$ position, being shallower on the left and deeper on the right, as shown in Figs. 7 and 8. Here the mean roll thickness $\overline{\delta_{\text {roll }}}$ along the $x$ direction is plotted as squares, and the roll thicknesses at the left end $(x=0)$ and right end $x=4 L$ are represented as left- and right-pointing triangles. Both $\psi_{\max }$ and $\overline{\delta_{\text {roll }}}$ increase with time, and these increasing trends can be approximated adopting least-squares 


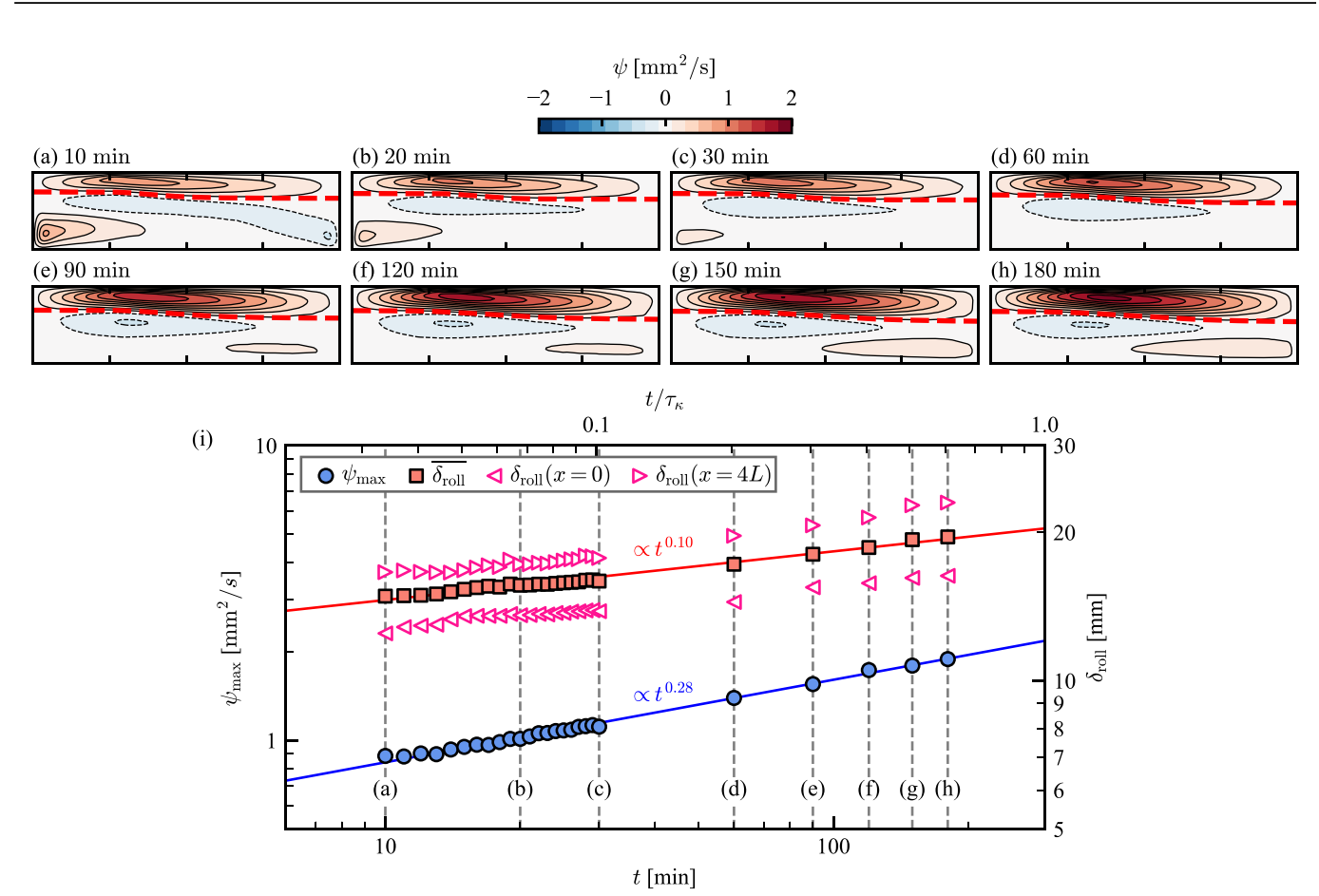

FIG. 9. Temporal evolution of roll structures at $t \geqslant 10$ min under the conditions $\Delta T=2 \mathrm{~K}$ and $\Delta \theta=2 \mathrm{~K}$ observed in (a)-(h) stream function fields and (i) characteristic values, $\psi_{\max }$ and $\overline{\delta_{\text {roll }}}$. Color contours shown in (a) to (h) represent the overall view of stream function fields and red dashed lines indicate the roll thickness $\delta_{\text {roll }}$. Circles and squares in (i) show changes in $\psi_{\max }$ and $\overline{\delta_{\text {roll }}}$ with time. Left- and right-pointing triangles are changes in $\delta_{\text {roll }}$ at $x=0$ and $x=4 L$, respectively. Blue and red solid lines are least-squares fits for $\psi_{\max }$ and $\delta_{\text {roll }}$. Vertical dashed lines in (i) correspond to the flow fields shown in (a) to (h).

fitting. In the case of the displayed condition, we obtain fitting curves of $\psi_{\max }=0.44 t^{0.28}$ and $\overline{\delta_{\text {roll }}}=11.6 t^{0.10}$ in dimensional units. For reference, the maximum horizontal velocity $u_{\max }$ increases with time with $u_{\max } \propto t^{0.14}$ for this condition, while this is not displayed in Fig. 9.

Under the condition of larger Ra, $\Delta T=2 \mathrm{~K}$ and $\Delta \theta=10 \mathrm{~K}$, the temporal evolution of the values are obtained as $u_{\max } \propto t^{0.03}, \psi_{\max } \propto t^{0.10}$, and $\overline{\delta_{\text {roll }}} \propto t^{0.10}$. The temporal dependencies of $u_{\max }$ and $\psi_{\max }$ are weaker than those in the case that $\Delta T=2 \mathrm{~K}$ and $\Delta \theta=2 \mathrm{~K}$, shown in Fig. 9. Considering that the roll thickness increases with the same temporal dependence, the roll thickness is not affected by the strength of the roll structures, and other factors may therefore be dominant in determining the roll thickness.

Mori and Niino [10] suggested self-similar solutions for determining the horizontal and vertical length scales of developing nonlinear $\mathrm{HC}$ in a laterally and vertically infinite system. The vertical length scale $\delta$ is given as the thermal diffusion length scale $\delta_{\kappa} \propto t^{1 / 2}$. The horizontal length scale $\ell$ is given as the integration of the speed of gravity current, $U_{\mathrm{gc}} \propto \sqrt{g \beta \Delta \theta \delta_{\kappa}} \propto t^{1 / 4}$, and thus $\ell \propto t^{5 / 4}$. Making the same assumptions adopted in Mori and Niino [10], the stream function also changes with time as $\psi \propto t^{3 / 4}$. Such self-similar solutions, however, may easily collapse in the case of a finite domain, and the length scale is immediately determined by the geometry as $\ell \sim 4 L$ in the present study. Thus, the temporal evolution of the two quantities, $\delta_{\text {roll }} \propto t^{0.10}$ and $\psi_{\max } \propto t^{0.28}$, may be slow compared to those obtained theoretically in the infinite system by Mori and Niino [10]. Such slow evolution may originate in the finite system, which potentially increases the effect of the restoring force of the stable stratification by forcing the flow directions. The temporal evolution of the roll structures thus may not have a universal trend even in the same finite domain of the fluid container. 


\section{B. Effect of stable temperature stratification}

For all the explored cases, the roll structures are confined in the upper regions as shown in Figs. 9(a)-9(h). Mullarney et al. [23] also showed a similar flow structure confined in a shallow region during their transient process to a thermally equilibrated state. This type of flow structure is superficially similar to the so-called boundary-layer regime in thermally equilibrated HC [13]; a shallow circulating region is formed owing to strong advection, and the circulation allows temperature variations only in the circulating region. The boundary-layer regime also allows presence of thermal plumes at the end of destabilizing surface, and thus isotherms are not fully closed within the circulating region but connected throughout the domain height. In this sense, the flow structures realized in this study are different from those in the boundary-layer regime, because the roll structures are always regulated by the restoring force of stable temperature stratification over the whole horizontal position, meaning isotherms closed within the roll region.

Since $\gamma_{\text {lin }}^{\prime}$ is considered as the primary factor, it is worth summarizing $\gamma_{\text {lin }}^{\prime}$ dependence of various flow characteristics. The maximum horizontal velocity $u_{\max }$, the maximum stream function $\psi_{\max }$, and the mean roll thickness $\overline{\delta_{\text {roll }}}$ are shown for all the explored parameters in Fig. 10. These characteristic quantities are plotted in $\gamma_{\text {lin }}^{\prime}$ domain as shown in Figs. 10(a), 10(c), and 10(e) and also plotted in the equivalent Ra domain as shown in Figs. 10(b), 10(d), and 10(f). These are shown for reference to compare the previous studies conducted in thermally equilibrated $\mathrm{HC}[1,5,13]$. Ra here simply represents the horizontal temperature difference $\Delta \theta$. Here all data recorded after $t=30 \mathrm{~min}$ are plotted so as not to include the first transient states. As found in Fig. 9, the flow structures develop with time during the measurements, and each condition thus has temporal deviations. The temporal evolution is indicated by blue arrows in Figs. 10(a), 10(c), and 10(e), while such evolution may not be apparent in Figs. 10(b), 10(d), and 10(f) as the plots are overlapping.

In Figs. 10(a) and 10(b), $u_{\max }$ for various explored parameters is plotted in comparison with $\gamma_{\text {lin }}^{\prime}$ or Ra. Each plot is colored by the values of Ra and $\gamma_{\text {lin }}^{\prime}$, respectively. The dependence of $u_{\max }$ on $\gamma_{\text {lin }}^{\prime}$ is shown in Fig. 10(a). Values of $u_{\max }$ in strongly stratified cases are lower than those in weakly stratified cases. The present system is horizontally finite, and the gravity current is forced to turn to the lower fluid layer against the stratification as shown in Fig. 6(c). This geometric confinement may augment the braking force of the stable temperature stratification, and $u_{\max }$ is considered to be smaller in the strongly stratified cases. In the case of fixed $\mathrm{Ra}(\Delta \theta)$ conditions, $u_{\max }$ has almost the same values especially under the conditions of larger Ra, regardless of the $\gamma_{\text {lin }}^{\prime}$ values. Decreasing in $\mathrm{Ra}$, in turn, means to approach to the gravity wave regime, and thus the $\gamma_{\text {lin }}^{\prime}$ dependence gradually becomes prominent with Ra decreasing. From these plots, the maximum horizontal velocity is found to be determined roughly by $\operatorname{Ra}(\Delta \theta)$ values, if the braking force of the stable stratification is weak compared with the advection induced by the baroclinic torque.

In Figs. 10(c) and 10(d), the maximum stream function $\psi_{\max }$ is plotted as functions of $\gamma_{\text {lin }}^{\prime}$ and $\mathrm{Ra}$. The right axes of the two plots are the maximum stream function $\psi_{\max }$ normalized by the thermal diffusion coefficient $\kappa$. The $\psi_{\max }$ values plotted in the $\gamma_{\text {lin }}^{\prime}$ domain [Fig. 10(c)] show clear dependence on the $\gamma_{\operatorname{lin}}^{\prime}$ for fixed Ra conditions. As in the example illustrated in Fig. 9(i), the values of $\psi_{\max }$ increase with time for each initial condition as indicated by the blue arrow. In contrast to relevant dependence on $\gamma_{\text {lin }}^{\prime}$ shown in Fig. 10(c), the plots are widely distributed in Fig. 10(d).

The mean roll thickness $\overline{\delta_{\text {roll }}}$ for the explored parameters is plotted in Figs. 10(e) and 10(f). In the case of the present parameters, the roll structures penetrated the fluid layer up to $\sim 50 \%$ of the total height $L$. For fixed Ra conditions, $\overline{\delta_{\text {roll }}}$ strongly depends on $\gamma_{\text {lin }}^{\prime}$ as shown in Fig. 10(e), while showing the growth with time under each initial condition is indicated by the blue arrow. Similar to the plots of $\psi_{\max }$ in Fig. 10(d), $\overline{\delta_{\text {roll }}}$ does not have a clear dependence on Ra as shown in Fig. 10(f).

Incorporating all the results shown in Fig. 10, the stratification parameter $\gamma_{\operatorname{lin}}^{\prime}$ seems to dominate the characteristic quantities of the developing HC. While $\gamma_{\text {lin }}^{\prime}$ has importance, the plots for each condition have temporal changes as shown by the blue arrows in Figs. 10(a), 10(c), and 10(e). The above discussion is based on constant stratification parameters $\gamma_{\text {lin }}^{\prime}$ defined in Eq. (7) using a fixed vertical temperature gradient $\gamma_{\text {lin }}$. Considering $\gamma_{\text {lin }}^{\prime}$ as constants may not be appropriate for 

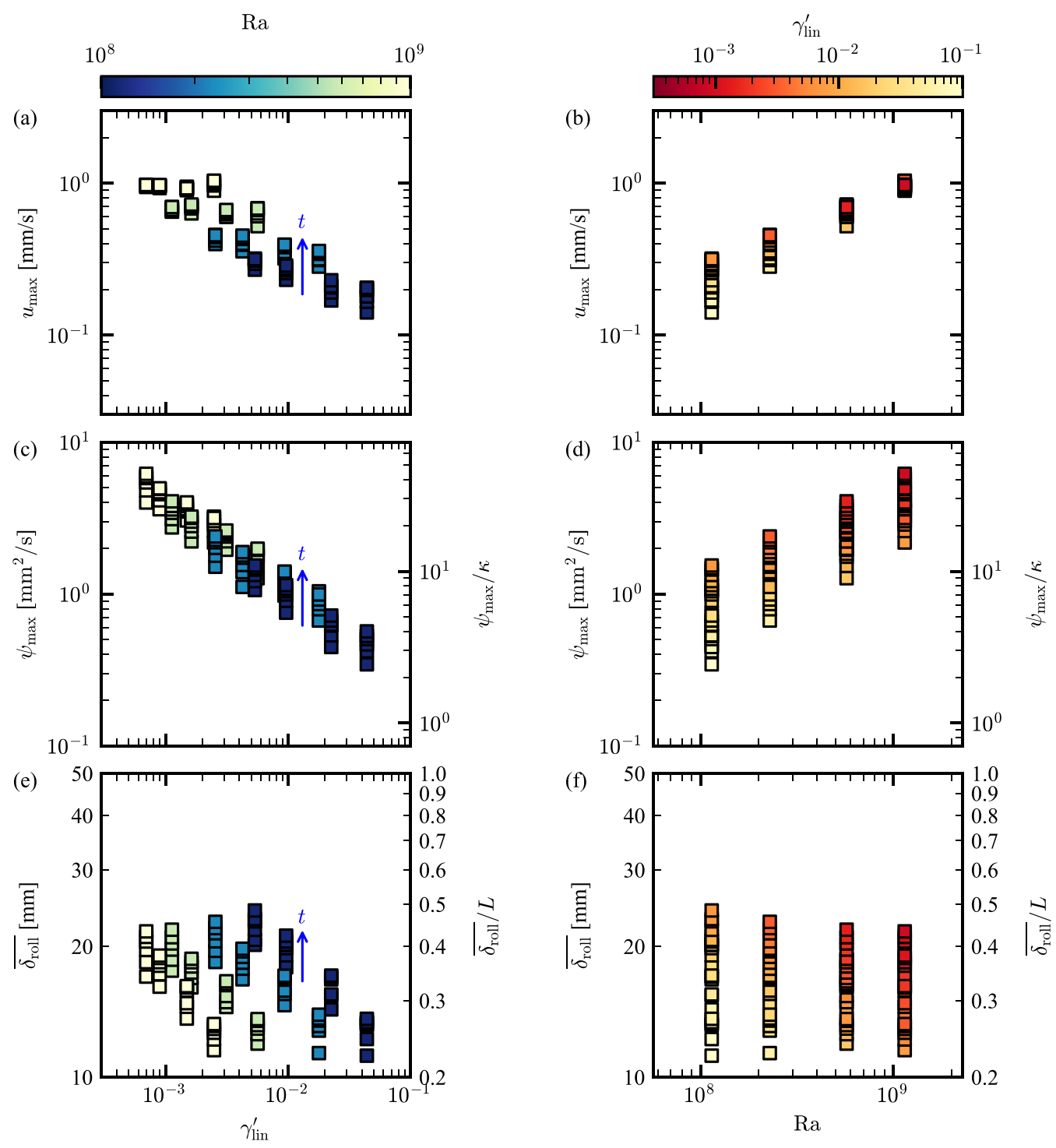

FIG. 10. Plots of characteristic values of horizontal convection for all the explored parameters measured at $t \geqslant 30$ min in Ra or $\gamma_{\text {lin }}^{\prime}$ domains: (a) and (b) the maximum horizontal velocity $u_{\max }$; (c) and (d) maximum stream function values; and (e) and (f) roll thickness $\overline{\delta_{\text {roll }}}$ averaged over the $x$ direction. Colors of plots in (a), (c), and (e) represent the Ra values while those in (b), (d), and (f) are the stratification parameter $\gamma_{\text {lin }}^{\prime}$. Blue arrows in (a), (c), and (e) guide the temporal evolution.

describing all the trends shown in Fig. 10, as $\gamma$ and $\gamma^{\prime}$ should originally be functions of both the horizontal coordinate $x$ and time $t$. In the present system, the fluid layer is always heated from the top and other walls are quasiadiabatic. Similarly, the stable temperature stratification relaxes, and $\gamma$ and $\gamma^{\prime}$ may change from $\infty$ to 0 with time, without equilibrium states being realized during the measurements. That is, the $\gamma^{\prime}$ characterizing the flow structure requires the consideration of the temporal evolution. This will be discussed in the following section. 


\section{Effect of relaxing stable temperature stratification}

As shown in Fig. 9, the roll structure and its characteristics change with time in developing HC. It is thus natural to replace the vertical length scale $L$ for the stratification parameter $\gamma_{\text {lin }}^{\prime}$ [Eq. (7)] by another length scale considering temporal evolution of the developing HC. Mori and Niino [10] explained this temporal evolution can be described together with the thermal diffusion from the top boundary, because the convective heat transport by the circulating motion is small. In the developing $\mathrm{HC}$, the vertical temperature gradient $\gamma$ relaxes with time owing to both convective heat transport and thermal diffusion from the heating copper blocks at the top. From the results shown in Fig. 6 and Figs. 10 (a) and 10 (b), the characteristic velocity scale is $O(0.1 \mathrm{~mm} / \mathrm{s})$. The horizontal dimension of the domain is $O(100 \mathrm{~mm})$, and the overturning timescale of the convective motions is thus estimated as $O\left(10^{3} \mathrm{~s}\right)$. This is comparable to or longer than the thermal diffusion timescale within the roll region $\tau_{\kappa, \text { roll }}=\delta_{\text {roll }}^{2} / \kappa=O\left(10^{2}-10^{3} \mathrm{~s}\right)$. These discussions can be justified in terms of the Péclet number, $\mathrm{Pe}=\psi / \kappa$. As shown in Figs. 10(c) and 10(d), the Pe defined using the maximum stream function $\psi_{\max }$ ranges from $\sim 2$ to $\sim 50$. Thus the Pe defined using the mean $\psi$ values within the roll region is $O\left(\leqslant 10^{0}\right)$, and this implies the less contribution of the convective transport on the heat transport compared to the diffusion. Accordingly, the thermal diffusion timescale may be dominant in the present setting, and the heating of the fluid layer is mainly carried out by thermal diffusion rather than convective heat transport.

Considering the discussion above, it is reasonable to include the relaxation process of the stable temperature stratification to the stratification parameter $\gamma^{\prime}$, in terms of the thermal diffusion. As the convective heat transport is trivial in this setting, the thermal diffusion process is supposed to be one-dimensional heating from the top; the fluid with initial temperature $T_{0}$ is heated to $T_{\text {wall }}>T_{0}$ from the top copper plate at $z=L$. The temperature profile along the $z$ axis can be regarded as an unsteady thermal conduction solution in the form

$$
T(z, t)=T_{0}+\left(T_{\text {wall }}-T_{0}\right) \operatorname{erfc}\left(\frac{L-z}{2 \sqrt{\kappa t}}\right),
$$

where erfc is the complementary error function. The vertical temperature gradient at the top is then derived as

$$
\left.\frac{\partial T}{\partial z}\right|_{z=L}=\frac{T_{\mathrm{wall}}-T_{0}}{\sqrt{\pi \kappa t}} \propto \frac{T_{\mathrm{wall}}-T_{0}}{\delta_{\kappa}} .
$$

By replacing the dimensional stratification parameter $\gamma$ with the vertical temperature gradient at the top [Eq. (16)] as $\gamma=\left(T_{\text {wall }}-T_{0}\right) / \delta_{\kappa}$, the nondimensional stratification parameter $\gamma^{\prime}$ with consideration of the temporal evolution can be defined. Here the temperatures at the top copper blocks differ as $T_{\text {wall }}=T_{0}+\Delta T+\Delta \theta(0 \leqslant x \lesssim L)$ and $T_{\text {wall }}=T_{0}+\Delta T(L \lesssim x \leqslant 4 L)$, and the mean wall temperature (6) is used. The mean vertical temperature gradient $\gamma$ is simply calculated similarly to the calculation in Eq. (7) as

$$
\gamma=\frac{4 \Delta T+\Delta \theta}{4 \sqrt{\kappa t}} .
$$

Accordingly, the nondimensional stratification parameter $\gamma^{\prime}$ considering temporal evolution is written as

$$
\gamma^{\prime}=\frac{g \beta(4 \Delta T+\Delta \theta)}{4 \sqrt{\kappa t}}\left(\frac{\sqrt{\kappa}}{g \beta \Delta \theta}\right)^{4 / 3},
$$

where the length scale $L$ in Eq. (8) is simply replaced by $\delta_{\kappa}$. We here consider that the degree of stable temperature stratification within the thermal boundary layer will be relaxed with $\gamma^{\prime} \propto t^{-1 / 2}$.

We now consider the flow characteristics shown in Fig. 10 again while considering the relaxing stable stratification. The various flow characteristics compared in $\gamma_{\operatorname{lin}}^{\prime}$ and Ra domains (Fig. 10) are 

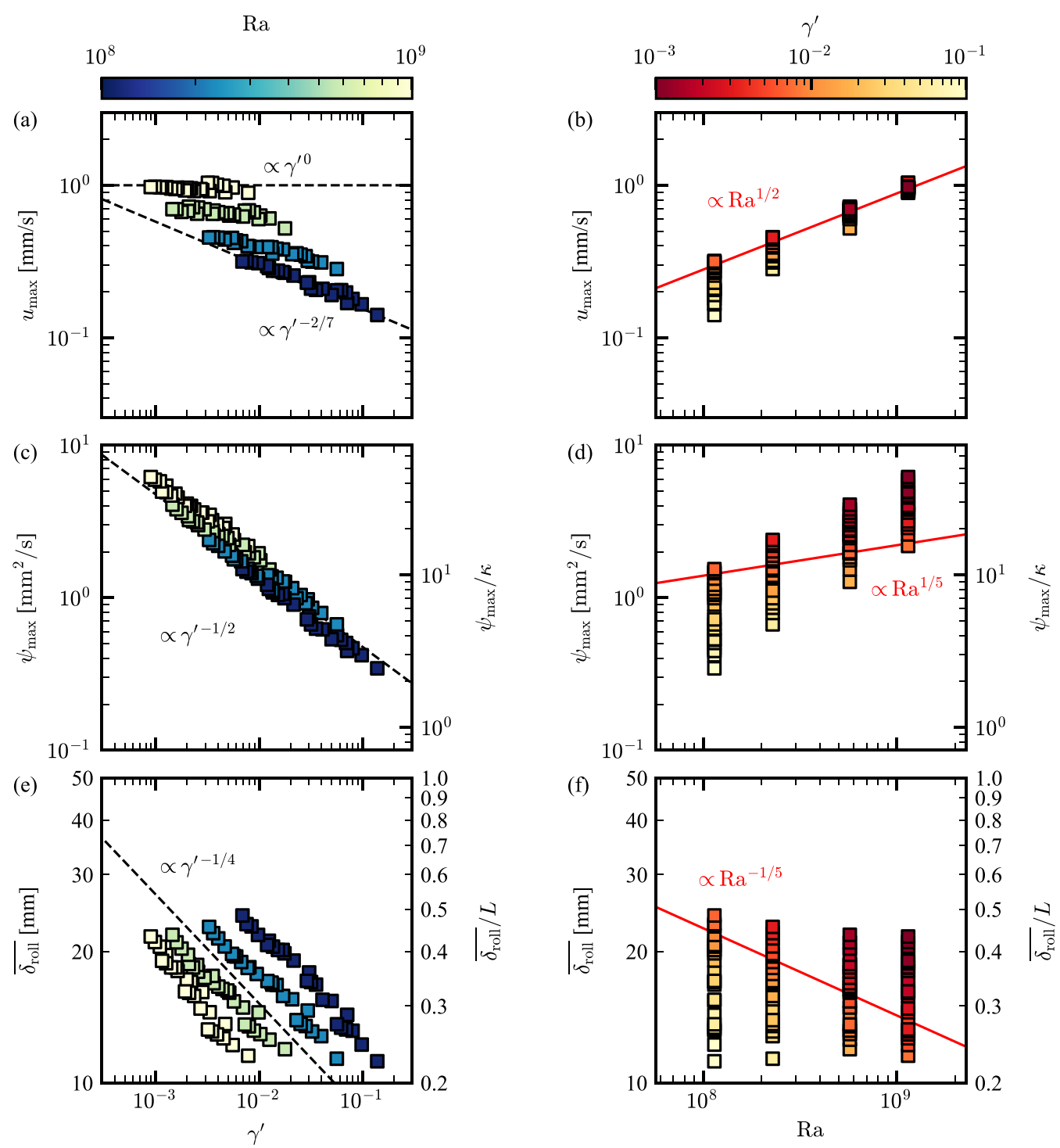

FIG. 11. Plots of characteristic values of horizontal convection for all the explored parameters after $t \geqslant 30$ min in $\mathrm{Ra}$ or $\gamma^{\prime}$ domains: (a) and (b) the maximum horizontal velocity $u_{\max }$; (c) and (d) maximum stream function values; (e) and (f) roll thickness $\overline{\delta_{\text {roll }}}$ averaged over the $x$ direction. Colors of plots in (a), (c), and (e) represent the Ra values while those in (b), (d), and (f) are the stratification parameter $\gamma^{\prime}$. Black dashed lines in (a), (c), and (e) are power laws that seemingly fit the present data, and red solid lines in (b), (d), and (f) are power laws predicted in previous studies.

replotted in $\gamma^{\prime}$ and Ra domains as shown in Fig. 11. In Figs. 11(a), 11(c), and 11(e) the horizontal axes give $\gamma^{\prime}$, the plots in Figs. 11(b), 11(d), and 11(f) are colored by $\gamma^{\prime}$.

As for the maximum horizontal velocity $u_{\max }$, plotted in Fig. 11(a), the temporal deviations, which exist in the $u_{\max }$ axis of Fig. 10(a), are no longer visible, and all the data for a fixed Ra value align along a single line. The $\gamma^{\prime}$ dependence can be approximated as $u_{\max } \propto \gamma^{\prime-2 / 7}$ in the cases of lowest $\mathrm{Ra}$, while the cases of largest Ra have almost constant $u_{\max }$, and these are indicated by the black dashed lines drawn in Fig. 11(a). In contrast, it may be difficult to find appreciable differences 
between the plots in Figs. 11(b) and 10(b). On the basis of the flow regimes characterized according to $\gamma^{\prime}[10]$, the case investigated in the present study is in the gravity current regime, $\gamma_{\text {lin }}^{\prime} \ll O(1)$. In this regime, the characteristic horizontal velocity can be estimated as the propagation speed of the gravity current, $U_{\mathrm{gc}}=\sqrt{g \beta \Delta \theta \delta_{\kappa}} \propto \Delta \theta^{1 / 2} \propto \mathrm{Ra}^{1 / 2}$, where the thermal diffusion length scale $\delta_{\kappa}=\sqrt{\kappa t}$ is considered [10,11]. This Ra dependence is shown as the red solid line in Fig. 11(b), and roughly matches the measured results.

The values of the maximum stream function $\psi_{\max }$ are shown in Figs. 11(c) and 11(d). The $\psi_{\max }$ plots in the $\gamma^{\prime}$ domain [Fig. 11(c)] are well organized regardless of the time elapsed from heating at the top, and the plots are aligned along a single line for each $\mathrm{Ra}$ condition. This line can be approximated as $\psi_{\max } \propto \gamma^{-1 / 2}$ as shown by the black dashed line in Fig. 11(c). In contrast, similar to the case for $u_{\max }$, differences in Fig. 11(d) compared with Fig. 10(d) are difficult to recognize. In the boundary-layer regime of thermally equilibrated $\mathrm{HC}$, it is possible to find a scaling for the maximum stream function as

$$
\psi_{\max } \sim \mathrm{Ra}^{1 / 5}
$$

and this scaling has been justified in many previous works $[1,5,13]$. In the developing $\mathrm{HC}$, the 1/5-power law [Eq. (19)] should not be useful, however, it is indicated for reference as the red solid line in Fig. 11(d). As expected, it is not possible to find certain scaling of Eq. (19).

The mean roll thicknesses $\overline{\delta_{\text {roll }}}$ are plotted in Figs. 11(e) and 11(f). The $\gamma^{\prime}$ dependence is clear for $\overline{\delta_{\text {roll }}}$ as shown in Fig. 11(e). Largely deviating plots due to the temporal evolution in Fig. 10(e) now lie on a line for each Ra condition. This $\gamma^{\prime}$ dependence of $\overline{\delta_{\text {roll }}}$ is approximated as $\overline{\delta_{\text {roll }}} \propto \gamma^{\prime-1 / 4}$ as indicated by the black dashed line in Fig. 11(e). In the boundary-layer regime, the scaling for the roll thickness is derived as

$$
\delta_{\text {roll }} \sim \mathrm{Ra}^{-1 / 5},
$$

together with Eq. (19) $[1,5,13]$. This scaling (20) is also not necessarily held in the developing HC, and the corresponding line is indicated for a reference by the red solid line in Fig. 11(f). For $\overline{\delta_{\text {roll }}}$, it is also not possible to find the Ra scaling previously claimed in thermally equilibrated HC [Eq. (20)].

Incorporating all the discussions above, the developing stratification parameter $\gamma^{\prime}$ defined in Eq. (18) describes well the flow characteristics of the developing HC for each Ra condition. Additionally, the braking force due to the stable stratification represented by $\gamma^{\prime}$ is considered to depend on the geometrical conditions and not only the temperature imposed at the top. If a horizontally smaller fluid container is employed while keeping the temperature difference at the top, the circulation distance will be shortened while the same baroclinic vorticity is produced. This condition will produce a braking force stronger than that in the case of the larger aspect ratio. It is therefore necessary to evaluate the roll structures using bulk characteristic values that consider the geometric environment.

\section{Bulk characteristics of developing horizontal convection}

To take the geometrical dependence into account, bulk quantities that consider the whole fluid layer may be good indicators with which to characterize the roll structures. Note that the present system can be regarded as two-dimensional flows, and the bulk quantities that will be discussed in the following are measured in the visualized cross section $0 \leqslant x \leqslant 4 L$ and $0 \leqslant z \leqslant L$ at $y=0.5 L$. The maximum stream function $\psi_{\max }$ represents the flow rate of the roll structure, which does not consider the geometric constraints, and this is not appropriate to characterize the strength of the roll structures. Instead of $\psi_{\max }$, we compute the circulation of the roll region $\Gamma_{\mathrm{c}}$ as

$$
\Gamma_{\mathrm{c}}=\int_{S} \boldsymbol{\omega} \cdot d \boldsymbol{S}=\int_{S_{\mathrm{roll}}} \eta d S,
$$


where $S_{\text {roll }}$ is the area of the closed roll region that has been determined using Eq. (14) and is calculated as

$$
S_{\text {roll }}=\int_{0}^{4 L} \delta_{\text {roll }}(x) d x=4 L \overline{\delta_{\text {roll }}}
$$

In considering the effect of the geometric environment, $\Gamma_{\mathrm{c}}$ may be more important than $\psi_{\max }$, since $\Gamma_{\mathrm{c}}$ can consider the strength of the rotation due to the roll structures originating in the baroclinic vorticity imposed at the top. Therefore, $\Gamma_{\mathrm{c}}$ is a better indicator with which to observe the balance of the baroclinic torque and the braking force of the stable stratification during the developing $\mathrm{HC}$. In Figs. 12(a) and 12(b), the circulation $\Gamma_{\mathrm{c}}$ is plotted as functions of $\gamma^{\prime}$ and Ra, respectively. A clear $\gamma^{\prime}$ dependence of $\Gamma_{\mathrm{c}}$ appears in Fig. 12(a) and is approximated as $\Gamma_{\mathrm{c}} \propto \gamma^{\prime-1 / 4}$; this is weaker than the $\gamma^{\prime}$ dependence of $\psi_{\max }\left(\psi_{\max } \propto \gamma^{\prime-1 / 2}\right)$. Under a fixed Ra condition, $\Gamma_{\mathrm{c}}$ is determined only by $\gamma^{\prime}$, similar to the case for $\psi_{\max }$ values shown in Fig. 11(c). Meanwhile, the $\Gamma_{\mathrm{c}}$ increases with $\mathrm{Ra}$ as shown in Fig. 12(b).

The flow fields shown in Figs. 7 and 9 suggest the existence of weak second and third roll structures beneath the first roll structure in the upper half of the fluid container. The layered second and third rolls are anticipated to be driven by the viscous force of the first roll structure, not directly by the baroclinic torque. Counterclockwise and clockwise circulations are thus generated in order beneath the first clockwise circulation. Similar flow structures were observed in the transient process from one thermally equilibrated HC to another when the destabilizing source was weakened [2,24]. Accordingly, the circulations of the second and third rolls are expected to be strong when the circulation of the first roll is strong. To simultaneously consider all the roll structures, the bulk total kinetic energy $\mathrm{KE}_{\mathrm{t}}$ is computed as

$$
\mathrm{KE}_{\mathrm{t}}=\int \frac{1}{2}\left(u^{2}+w^{2}\right) d A=\int_{0}^{L} \int_{0}^{4 L} \frac{1}{2}\left(u^{2}+w^{2}\right) d x d z
$$

where $\mathrm{KE}_{\mathrm{t}}$ has a unit of $\mathrm{mm}^{4} / \mathrm{s}^{2}$. In Figs. 12(c) and 12(d), $\mathrm{KE}_{\mathrm{t}}$ values are plotted as functions of $\gamma^{\prime}$ and Ra, respectively. The $\gamma^{\prime}$ dependence appears clearly as shown in Fig. 12(c), suggesting a scaling of $\mathrm{KE}_{\mathrm{t}} \propto \gamma^{\prime-5 / 7}$ as drawn by the black dashed line. As expected, conditions of larger $\mathrm{Ra}$ result in larger $\mathrm{KE}_{\mathrm{t}}$ values overall in Fig. 12(d).

In addition to the total kinetic energy $\mathrm{KE}_{\mathrm{t}}$, the Reynolds number Re may be another important quantity. It is defined as $\operatorname{Re}=U_{\mathrm{rms}} L / v$, where $U_{\text {rms }}$ is the root-mean-squared velocity computed as

$$
U_{\mathrm{rms}}=\sqrt{\frac{\mathrm{KE}_{\mathrm{t}}}{4 L^{2}}} .
$$

In Figs. 12(e) and 12(f), $U_{\text {rms }}(\mathrm{Re})$ values are plotted as functions of $\gamma^{\prime}$ and Ra. The computed Re values are distributed in the range of $O\left(10^{0}-10^{1}\right)$. As the Re values are in the relation $\operatorname{Re} \propto \sqrt{\mathrm{KE}_{\mathrm{t}}}$, the overall trends are similar to those in Figs. 12(c) and 12(d), and $\gamma^{\prime}$ dependency appears as $\operatorname{Re} \propto$ $\gamma^{\prime-5 / 14}$, as shown by the black dashed lines.

The bulk quantities shown in Fig. 12 also suggest dependencies on $\gamma^{\prime}$, similar to the characteristic quantities shown in Fig. 11. Details of these parameter dependencies are discussed in the following section.

\section{DISCUSSION}

In summarizing the results presented in Sec. III, the various characteristic quantities of developing HC shown in Figs. 11 and 12 seem to depend on $\gamma^{\prime}$. While the presence of destabilizing source is not allowed in the present study, it may be possible to find Ra dependence of these characteristic quantities, because Ra represents strength of baroclinic torque. Assuming that these dependencies can be expressed by certain power law relations like Eqs. (19) and (20), we have

$$
\phi \propto \gamma^{\prime p} \mathrm{Ra}^{q}
$$



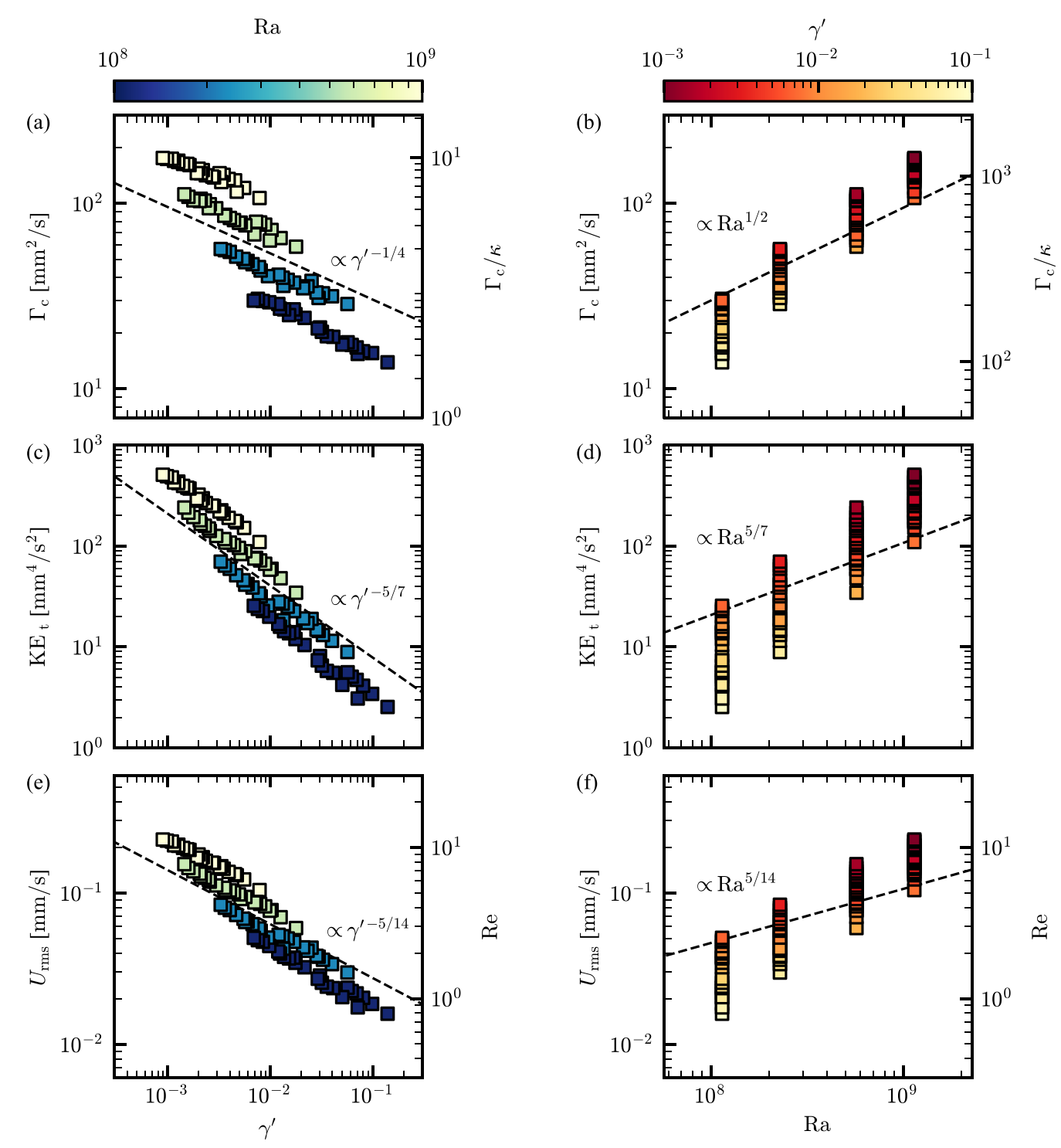

FIG. 12. Plots of bulk representative values of horizontal convection after $t \geqslant 30 \mathrm{~min}$ as functions of Ra and $\gamma^{\prime}$ : (a) and (b) the circulation of the roll region $\Gamma_{\mathrm{c}}$; (c) and (d) total kinetic energy $\mathrm{KE}_{\mathrm{t}}$; and (e) and (f) root-mean-squared velocity $U_{\text {rms }}$ (left axis) and Reynolds number Re (right axis). Colors of plots in (a), (c), and (e) represent the Ra values, while those in (b), (d), and (f) are the stratification parameter $\gamma^{\prime}$. Black dashed lines in (a), (c), and (e) are power laws of $\gamma^{\prime}$, while those in (b), (d), and (f) are power laws of Ra, seemingly fitting the present data.

where $\phi$ takes any characteristic quantities that are plotted in Figs. 11 and 12, and $p$ and $q$ are, respectively, arbitrary scaling factors for $\gamma^{\prime}$ and Ra. The optimal scaling factors are simultaneously computed for the measured quantities shown in Figs. 11 and 12 adopting singular value decomposition (SVD). Note that Ra considered here does not represent the buoyancy produced at the destabilizing source, and represents the strength of baroclinic torque alone. In Fig. 13 the characteristic quantities (a) $u_{\max }$, (b) $\psi_{\max }$, (c) $\overline{\delta_{\text {roll }}}$, (d) $\Gamma_{\mathrm{c}}$, (e) $\mathrm{KE}_{\mathrm{t}}$, and (f) Re are plotted as functions of both $\gamma^{\prime}$ and Ra. The colors of the plots represent the measured quantities shown in the color 

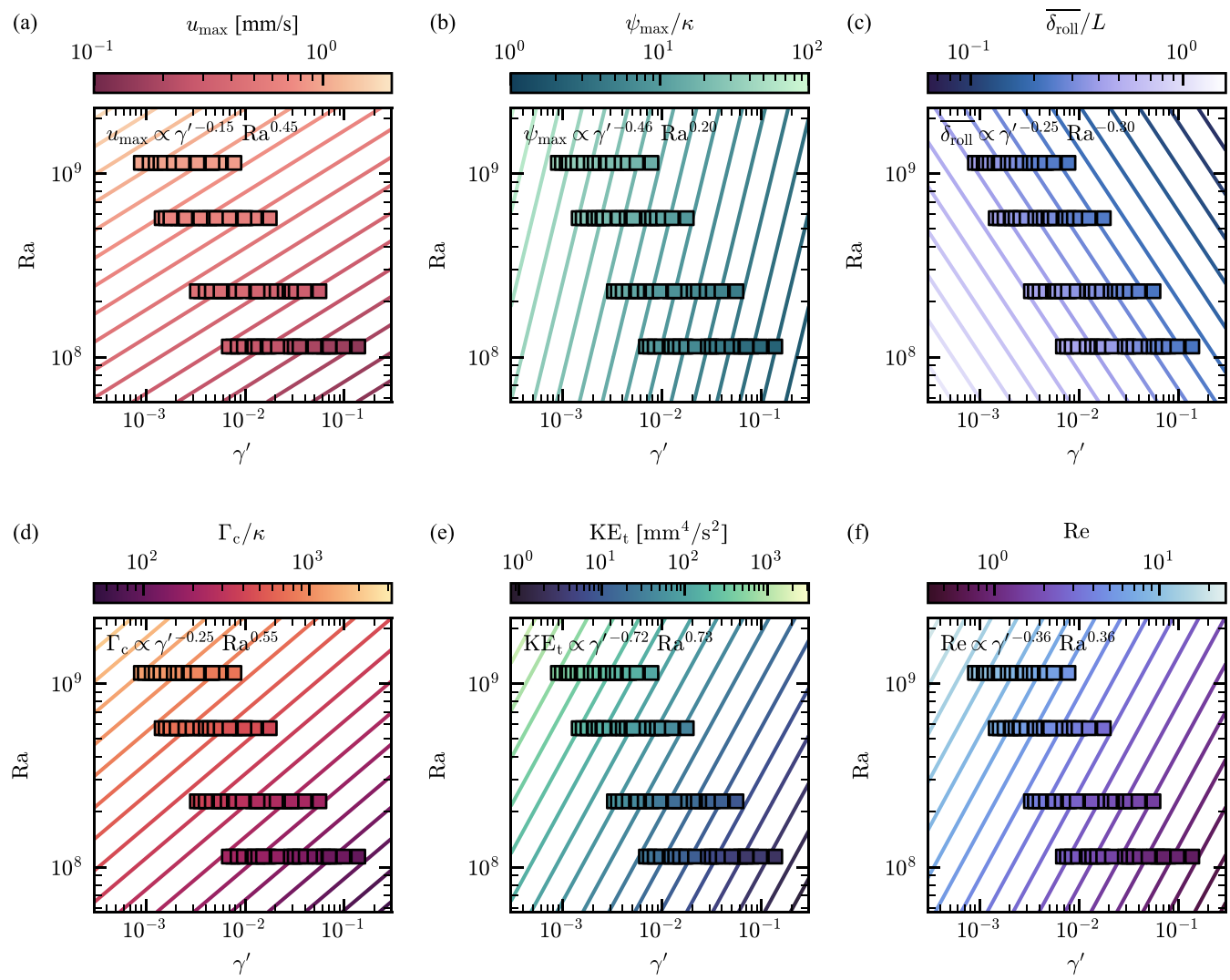

FIG. 13. Various quantities plotted in the $\gamma^{\prime}$-Ra domain; (a) maximum horizontal velocity $u_{\max }$, (b) maximum stream function $\psi_{\max }$, (c) average roll thickness $\overline{\delta_{\text {roll }}}$, (d) circulation of the roll region $\Gamma_{\mathrm{c}}$, (e) total kinetic energy $\mathrm{KE}_{\mathrm{t}}$, and (f) the Reynolds number Re. Colors of the plots represent the quantities shown above each panel. Colored contour lines shown behind the plots are the planes fitted in the SVD method, and the scaling factors computed in the method are noted at the upper left of each panel.

bar above each panel. The colored contour lines drawn behind the plots are the optimal planes determined adopting the SVD method, and the scaling factors are noted on each panel. Overall, the colors of the plots that show the measured values are similar to those of the computed planes, and this suggests that the six quantities shown in Fig. 13 can be represented by the power laws for the two nondimensional parameters $\gamma^{\prime}$ and Ra. Large $\gamma^{\prime}$ values represent a strong restoring force of the stable temperature stratification against the baroclinic circulation, and large Ra values mean that strong baroclinic vorticities are imposed on the fluid layer.

The scaling for $u_{\max }$ is computed as $u_{\max } \propto \gamma^{\prime-0.15} \mathrm{Ra}^{0.45}$, as suggested in Fig. 13(a). The $u_{\max }$ values have a weak dependence on $\gamma^{\prime}$. The scaling factor for Ra can be considered to represent the $1 / 2$-power law that may originate from the propagation speed of the gravity current $U_{\mathrm{gc}} \propto \mathrm{Ra}^{1 / 2} \propto$ $\Delta \theta^{1 / 2}$ as discussed in Sec. III B. From the $\gamma^{\prime}$ dependence of $u_{\max }$, the present flow regime can be considered to lie between the gravity current and the gravity wave regimes [10] even though $\gamma^{\prime}$ is sufficiently small. In general, the gravity wave regime appears when the restoring force of the stable temperature stratification is much stronger than the baroclinic torque. For the smallest $\Delta \theta, \Delta \theta=1 \mathrm{~K}$, the gravity wave regime requires $\Delta T \sim 250 \mathrm{~K}$ to be achieved with $\gamma^{\prime} \sim 1$, and of course, this is not realistic. That is, the finite domain of the fluid container increases the apparent restoring force by restricting the flow direction as discussed in Sec. III A. 
As shown in Fig. 13(b), the optimal scaling for $\psi_{\max }$ is found to be $\psi_{\max } \propto \gamma^{\prime-0.46} \mathrm{Ra}^{0.20}$. A strong $\gamma^{\prime}$ dependence is found to be $\gamma^{\prime} \sim-1 / 2$. Wang and Huang [4] showed the scaling, $\psi_{\max } \propto \mathrm{Ra}_{\delta}^{1 / 2}$ by defining the Rayleigh number within the velocity boundary layer $\mathrm{Ra}_{\delta}$. The thickness of the boundary layer is, however, not a predetermined value and is determined by the flow structure in a thermally equilibrated HC. Thus, they claimed that the layer thickness was difficult to use in practice. This 1/2-power law found in Wang and Huang [4] seems to correspond to the presently obtained scaling $\psi_{\max } \propto \gamma^{\prime-1 / 2}$, as the thickness of the velocity boundary layer $\delta$ can be considered to well represent the braking force due to the stable temperature stratification; $\delta$ becomes thicker as $\gamma^{\prime}$ becomes smaller. A point of this scaling is that the previously suggested scaling of $\psi_{\max } \propto \mathrm{Ra}^{1 / 5}$ in thermally equilibrated HC [Eq. (19)] is found even in the developing HC. The maximum stream function $\psi_{\max }$ is just a local maximum representing the strength of circulation induced by the baroclinic torque, and thus this may be determined by only the horizontal temperature difference $\Delta \theta$. The traditional scaling in Eq. (19) is therefore universal in the developing HC.

The scaling for $\overline{\delta_{\text {roll }}}$ is obtained as $\overline{\delta_{\text {roll }}} \propto \gamma^{\prime-0.25} \mathrm{Ra}^{-0.30}$ [Fig. 13(c)]. Unlike the case of $\psi_{\text {max }}$, the roll thickness is strongly affected by the restoring force of the stable temperature stratification and the geometric environment in the developing HC. This is because the roll structure can penetrate only the stratified fluid relaxed together with the thermal diffusion. The $\gamma^{\prime}$ dependence appears as $\sim-1 / 4$, and $\gamma^{\prime}$ seems to be important in determining the roll thickness. The roll thickness decreases for $\gamma^{\prime}$ values meaning strongly stratified situations. The scaling factor for Ra indicates a Ra dependence slightly stronger than the suggested one, $\delta_{\text {roll }} \propto \mathrm{Ra}^{-1 / 5}$ [Eq. (20)]; however, a similar decreasing trend is obtained. As the destabilizing source does not exist, Ra represents only the strength of baroclinic torque. The scaling in Eq. (20) may therefore not be necessarily acquired in the developing $\mathrm{HC}$.

In Fig. 13(d), $\Gamma_{\mathrm{c}}$ values are plotted, and the scaling factors are computed as $\Gamma_{\mathrm{c}} \propto \gamma^{\prime-0.25} \mathrm{Ra}^{0.55}$. The $\gamma^{\prime}$ dependence appears as a power to $\sim-1 / 4$. The circulation may be strongly affected by geometric confinements like that of the aspect ratio. However, it becomes large with a decrease in $\gamma^{\prime}$, similar to the case for $\psi_{\max }$. On the basis of Eqs. (21) and (22), the total vorticity produced in the roll region $\eta_{\text {roll }}$ is expressed as

$$
\eta_{\text {roll }}=\frac{1}{S_{\text {roll }}} \int_{S_{\text {roll }}} \eta d S=\frac{\Gamma_{\mathrm{c}}}{S_{\text {roll }}}=\frac{\Gamma_{\mathrm{c}}}{4 L \overline{\delta_{\text {roll }}}}
$$

Using the scalings computed in Figs. 13(c) and 13(d), the scaling for $\eta_{\text {roll }}$ is obtained as $\eta_{\text {roll }} \propto$ $\gamma^{10.00} \mathrm{Ra}^{0.85}$. This indicates the total vorticity produced by the horizontal temperature difference is determined only by $\mathrm{Ra}$ with no contribution made by $\gamma^{\prime}$. This result is consistent with the temperature gradient profiles shown in Fig. 5(b), in that the baroclinic vorticity production is determined only by the horizontal temperature difference $\Delta \theta \propto \mathrm{Ra}$. The exponent 0.85 is anticipated to asymptote to unity if the system is in full equilibrium like in the case of conventionally studied horizontal convection or there is no dissipation effect due to stable stratification and the finite fluid container.

The total kinetic energy $\mathrm{KE}_{\mathrm{t}}$ and $\mathrm{Re}$ as functions of $\gamma^{\prime}$ and Ra are shown in Figs. 13(e) and 13(f), respectively. For $\mathrm{KE}_{\mathrm{t}}$, the optimal scaling factors are obtained as $\mathrm{KE}_{\mathrm{t}} \propto \gamma^{\prime-0.72} \mathrm{Ra}^{0.73}$. This scaling suggests that the restoring force of the stable stratification works at the same degree of the baroclinic torque. The restoring force acting against the baroclinic torque suppresses the enhancement of the circulating motions, as well as the viscous dissipation. In addition, because the finite fluid container may augment the restoring force as the main flow structure, the first roll formed beneath the copper blocks is confined in a shallow region. The lower quiescent fluid is thus driven by the first roll owing to the fluid viscosity and forms the second and third rolls [2,24]. This viscous dissipation may appear as the negative dependence of $\mathrm{KE}_{\mathrm{t}}$ on $\gamma^{\prime}$. As the Re is proportional to the square of $\mathrm{KE}_{\mathrm{t}}$, the exponents for Re become half those for $\mathrm{KE}_{\mathrm{t}}$; these are computed as $\operatorname{Re} \propto \gamma^{\prime-0.36} \mathrm{Ra}^{0.36}$. From these exponents, the total kinetic energy, as well as the Reynolds number, becomes large when 
the imposed baroclinic torque is strong and the restoring force of the stable temperature stratification is weak.

The $\gamma^{\prime}$ dependencies of these characteristic quantities are modified by giving different geometrical conditions. As discussed in Sec. III A, the laterally finite domain regulates the circulating motion of the roll structures, and the restoring force of the stable temperature stratification strengthens as a result. This augmentation of the apparent restoring force may be prominent when a fluid container having a small aspect ratio is used for the fluid container. In this case, the $\gamma^{\prime}$ dependencies increase from those in the present case shown in Fig. 13. Meanwhile, a fluid container having a large aspect ratio or being laterally infinite may reduce the effect of the stable temperature stratification, and the $\gamma^{\prime}$ dependencies also decrease. When the $\gamma^{\prime}$ dependence becomes negligibly small, the developing $\mathrm{HC}$ is characterized only by Ra like in the case of thermally equilibrated $\mathrm{HC}$.

\section{CONCLUSIONS}

Transient process of developing horizontal convection $(\mathrm{HC})$, in which baroclinic torque is the primary factor determining flow structures, was investigated experimentally in detail. Unlike thermally equilibrated $\mathrm{HC}$, which has been studied previously, the developing HC excludes the presence of "destabilizing source" at the boundary. Water was used as the test fluid and a differential horizontal heating was imposed at the top of a quiescent cold water confined in a rectangular fluid container. Seeding of particles of thermochromic liquid crystals allowed optical visualization for the purpose of PTV. As destabilizing source does not exist, the flow structures were determined by only the balance of baroclinic torque and restoring force of stable temperature stratification. We therefore defined the nondimensional stratification parameter $\gamma^{\prime}$, which expresses the restoring force of the stable temperature stratification acting against the baroclinic torque, apart from Rayleigh number which may be dominant in thermally equilibrated $\mathrm{HC}$.

During the developing stage of HC, the baroclinic torque becomes dominant due to the absence of destabilizing source. A single convection roll develops within a shallow region immediately beneath the heating boundary, and its motion was very slow meaning small contribution of convective heat transport. The convection roll grows with time due to the relaxation of stable temperature stratification by the thermal diffusion; the roll structure gradually penetrate the relaxed stratified fluid deeper with time. The temporal evolution and $\gamma^{\prime}$ or Ra dependencies of the flow structures, in terms of maximum velocity, maximum stream function, roll thickness, circulation, total kinetic energy, and Reynolds number, were evaluated for the flow fields. The nondimensional stratification parameter $\gamma^{\prime}$, considering the thermal diffusion, was a good indicator to represent the flow characteristics. For the explored parameter ranges, $\gamma^{\prime}=O\left(10^{-4}-10^{-2}\right)$ and $\mathrm{Ra}=O\left(10^{8}-10^{9}\right)$, the flow characteristics were well described by power laws in the form of $\gamma^{\prime p} \mathrm{Ra}^{q}$, and the scaling factors were determined for each measured quantity. The characteristic quantities showed relevant dependence on $\gamma^{\prime}$, even $\gamma^{\prime}$ changed with time together with the relaxing of the stable temperature stratification. In addition, the finite domain of the fluid container seems to augment the effect of the restoring force. The bulk quantities of the roll structures are suppressed by stable temperature stratification. These results suggest that the balance of the restoring force of stable temperature stratification and the baroclinic torque is a primary factor characterizing temporally developing $\mathrm{HC}$, while this may not be apparent in thermally equilibrated $\mathrm{HC}$.

\section{ACKNOWLEDGMENTS}

The authors acknowledge financial support obtained through a Grant-in-Aid for JSPS Fellows (Grant No. JP19J20096) and Fukada Grant-in-Aid from the Fukada Geological Institute.

[1] H. T. Rossby, On thermal convection driven by non-uniform heating from below: An experimental study, Deep Sea Research and Oceanographic Abstracts 12, 9 (1965). 
[2] T. Rossby, Numerical experiments with a fluid heated non-uniformly from below, Tellus A 50, 242 (1998).

[3] C. Wunsch and R. Ferrari, Vertical mixing, energy, and the general circulation of the oceans, Annu. Rev. Fluid Mech. 36, 281 (2004).

[4] W. Wang and R. X. Huang, An experimental study on thermal circulation driven by horizontal differential heating, J. Fluid Mech. 540, 49 (2005).

[5] M. ILIcak and G. K. Vallis,Simulations and scaling of horizontal convection, Tellus A 64, 18377 (2012).

[6] B. Gayen, R. Griffiths, and G. Hughes, Stability transitions and turbulence in horizontal convection, J. Fluid Mech. 751, 698 (2014).

[7] N. A. Vinnichenko, A. V. Pushtaev, Y. Y. Plaksina, Y. K. Rudenko, and A. V. Uvarov, Horizontal convection driven by nonuniform radiative heating in liquids with different surface behavior, Int. J. Heat Mass Transf. 126, 400 (2018).

[8] J. Lu, S. P. Arya, W. H. Snyder, and R. E. Lawson Jr, A laboratory study of the urban heat island in a calm and stably stratified environment. Part I: Temperature field, J. Appl. Meteorol. 36, 1377 (1997).

[9] J. Lu, S. P. Arya, W. H. Snyder, and R. E. Lawson Jr, A laboratory study of the urban heat island in a calm and stably stratified environment. Part II: Velocity field, J. Appl. Meteorol. 36, 1392 (1997).

[10] A. Mori and H. Niino, Time evolution of nonlinear horizontal convection: Its flow regimes and self-similar solutions, J. Atmos. Sci. 59, 1841 (2002).

[11] H. Niino, A. Mori, T. Satomura, and S. Akiba, Flow regimes of nonlinear heat island circulation, J. Atmos. Sci. 63, 1538 (2006).

[12] H. Gramberg, P. Howell, and J. Ockendon, Convection by a horizontal thermal gradient, J. Fluid Mech. 586, 41 (2007).

[13] S. Chiu-Webster, E. Hinch, and J. Lister, Very viscous horizontal convection, J. Fluid Mech. 611, 395 (2008).

[14] G. O. Hughes and R. W. Griffiths, Horizontal convection, Annu. Rev. Fluid Mech. 40, 185 (2008).

[15] O. Shishkina and S. Wagner, Prandtl-Number Dependence of Heat Transport in Laminar Horizontal Convection, Phys. Rev. Lett. 116, 024302 (2016).

[16] C. S. Vila, S. Discetti, G. M. Carlomagno, T. Astarita, and A. Ianiro, On the onset of horizontal convection, Int. J. Therm. Sci. 110, 96 (2016).

[17] O. Shishkina, Mean flow structure in horizontal convection, J. Fluid Mech. 812, 525 (2017).

[18] M. G. Rosevear, B. Gayen, and R. W. Griffiths, Turbulent horizontal convection under spatially periodic forcing: A regime governed by interior inertia, J. Fluid Mech. 831, 491 (2017).

[19] S. Hossain, T. Vo, and G. J. Sheard, Horizontal convection in shallow enclosures scales with height, not length, at low Rayleigh numbers, Int. Commun. Heat Mass 109, 104308 (2019).

[20] F. Paparella and W. Young, Horizontal convection is non-turbulent, J. Fluid Mech. 466, 205 (2002).

[21] A. Scotti and B. White, Is horizontal convection really non-turbulent, Geophys. Res. Lett. 38, L21609 (2011).

[22] M. A. Coman, R. W. Griffiths, and G. O. Hughes, Sandström's experiments revisited, J. Mar. Res. 64, 783 (2006).

[23] J. C. Mullarney, R. W. Griffiths, and G. O. Hughes, Convection driven by differential heating at a horizontal boundary, J. Fluid Mech. 516, 181 (2004).

[24] R. W. Griffiths, G. O. Hughes, and B. Gayen, Horizontal convection dynamics: Insights from transient adjustment, J. Fluid Mech. 726, 559 (2013).

[25] S. Anders, D. Noto, Y. Tasaka, and S. Eckert, Simultaneous optical measurement of temperature and velocity fields in solidifying liquids, Exp. Fluids 61, 1 (2020).

[26] D. Noto, Y. Tasaka, T. Yanagisawa, and T. Miyagoshi, Stepwise transitions in spin-up of rotating Rayleigh-Bénard convection, J. Fluid Mech. 911, A43 (2021).

[27] D. Shepard, A two-dimensional interpolation function for irregularly-spaced data, in Proceedings of the 1968 23rd ACM National Conference (Association for Computing Machinery, New York, 1968), pp. 517-524.

[28] K. Fujita, Y. Tasaka, T. Yanagisawa, D. Noto, and Y. Murai, Three-dimensional visualization of columnar vortices in rotating Rayleigh-Bénard convection, J. Vis. 23, 635 (2020). 